NBER WORKING PAPER SERIES

\title{
THE BEHAVIOR OF SAVINGS AND ASSET PRICES WHEN PREFERENCES AND BELIEFS ARE HETEROGENEOUS
}

\author{
Ngoc-Khanh Tran \\ Richard J. Zeckhauser \\ Working Paper 17199 \\ http://www.nber.org/papers/w17199
NATIONAL BUREAU OF ECONOMIC RESEARCH
1050 Massachusetts Avenue
Cambridge, MA 02138
July 2011

We are very grateful to Hui Chen, Scott Joslin, Leonid Kogan, Anh Tran, Raman Uppal and Jessica Wachter for many insightful discussions and suggestions. We are indebted to Jerome Detemple for his introduction to and lectures on Malliavin calculus. All the remaining errors are our own. The views expressed herein are those of the authors and do not necessarily reflect the views of the National Bureau of Economic Research.

NBER working papers are circulated for discussion and comment purposes. They have not been peerreviewed or been subject to the review by the NBER Board of Directors that accompanies official NBER publications.

(C) 2011 by Ngoc-Khanh Tran and Richard J. Zeckhauser. All rights reserved. Short sections of text, not to exceed two paragraphs, may be quoted without explicit permission provided that full credit, including $\odot$ notice, is given to the source. 
The Behavior of Savings and Asset Prices When Preferences and Beliefs are Heterogeneous Ngoc-Khanh Tran and Richard J. Zeckhauser

NBER Working Paper No. 17199

July 2011

JEL No. D51,G12

\begin{abstract}
$\underline{\text { ABSTRACT }}$
Movements in asset prices are a major risk confronting individuals. This paper establishes new asset pricing results when agents differ in risk preference, time preference and/or expectations. It shows that risk tolerance is a critical concept driving savings decisions, consumption allocations, prices and return volatilities. Surprisingly, due to the equilibrium risk sharing, the precautionary savings motive in the aggregate can vastly exceed that of even the most prudent actual agent in the economy. Consequently, a low real interest rate, resulting from large aggregate savings, can prevail with reasonable risk aversions for all agents. One downside of a large aggregate savings motive is that savings rates become extremely sensitive to output fluctuation. Thus, the same mechanism that produces realistically low interest rates tends to make them unrealistically volatile.
\end{abstract}

A powerful isomorphism allows differences in time preference and expectations to be swept away in the analysis, yielding an equivalent economy whose agents differ merely in risk aversion. These results hold great potential to simplify the analysis of heterogeneous-agent economies, as we demonstrate in quantifying how asset prices move and bounding their volatilities. All results are obtained in closed form for any number of agents possessing additively separable preferences in an endowment economy.

Ngoc-Khanh Tran

Massachusetts Institute of Technology

8 Sixth Street

Apt. 2

Cambridge, MA 02141

khanh@MIT.EDU

Richard J. Zeckhauser

John F. Kennedy School of Government

Harvard University

79 John F. Kennedy Street

Cambridge, MA 02138

and NBER

richard_zeckhauser@harvard.edu 


\section{Introduction}

The genius of the market is its ability to transform the holdings of agents with heterogeneous preferences and endowments into outcomes that are superior for all. When time and subjective beliefs enter the picture, agents' claims shift across time and state in patterns that reflect both aggregate shocks and their beliefs, and time and risk preferences. Aggregate measures in the economy, such as interest rates and saving rates, reflect the outcome of agents who trade within such dynamic market processes.

We assume, as is common in the consumption-based equilibrium asset pricing literature, that agents start with birthright endowments of a risky asset, i.e., the contingent claim on its stochastic dividend stream. The dividend is interchangeably referred to as endowment, output or supply hereafter. In addition there is a riskless asset created by the agents of zero net supply. The price of the risky asset and the interest rate are determined by the supply and demand of the market participants. Those participants possess additively separable utility functions. As the world unfolds, they allocate their available funds - asset values plus asset returns - among consumption and holdings of the two types of assets so as to maximize their discounted expected utility. Thus agents continually shift their portfolios as asset prices rise and fall in response to the economy (endowment). Such shifting would not take place if agents held identical preferences. Note agents are better off in this heterogeneous world. They could mimic a homogeneous world by just refusing to trade.

Our attention to heterogeneity in preferences is intended to capture real world richness, and to study the evolving patterns when diverse agents interact. Most prior analyses have eschewed heterogeneity, thereby sacrificing relevance to escape the technical intractability that normally accompanies attempts to allow for significant agent differences. We were able to define a new but straightforward construct that characterizes the dynamic contribution of individual agents to the demand for assets, and also identifies how current asset returns influence agents' optimal allocations.

We build on our analysis of differences in preferences to examine how disparate subjective beliefs about the economy's uncertain fundamentals also affect outcomes. Whatever the sources of differences, the risk-averse agents share the unavoidably variable aggregate output in a manner that smooths out their personal consumptions. Naturally, more risk averse and impatient consumers 
respectively get smoother and earlier consumption, but they get less and ultimately much less later consumption.

All of our results are obtained in closed form. We show that all aggregate quantities of interest can be expressed as functions of agents' equilibrium consumptions, which in turn respond to those aggregates. Agents whose consumptions are most sensitive to shocks, not surprisingly, contribute predominantly to influence the behavior of the economy as output fluctuates.

The risk tolerance measure that we advocate in the current paper captures this intuition of risk-sharing mechanism. It is defined as individual $i$ 's marginal propensity $\left\{\frac{\partial c^{i}}{\partial w}\right\}_{i}$ to consume $c^{i}$ out of the aggregate endowment $w$. It is proportional to individual risk tolerance, and shows that more risk tolerant agents embrace more volatile consumption paths (i.e., larger response of $\frac{\partial c^{i}}{\partial w}$ to an output shock) in return for greater shares of the endowment when times are good. It proves both convenient and reassuring that the economy's implied aggregate (i.e., market-revealed) behavior toward uncertainty, such as the risk premium and precautionary savings behavior inferred from the market prices, and the volatilities of its bond and stock returns can be readily expressed in terms of means and variances under this measure. For this reason, throughout this paper we will interchangeably refer to these aggregate behaviors as market-revealed, and market-equivalent characteristics of a fictitious equivalent single individual representing the entire body of agents. This aggregation is feasible in our complete-market economy.

In the special case of heterogeneous CRRA agents, it is well known that aggregate risk aversion decreases with aggregate consumption. Similarly, given that more risk-tolerant agents invest relatively more in the risky stock, a positive shock boosts their relative position in the economy, thereby making them more influential. Observed risk tolerance thus increases in good times, and vice versa, due to ownership shifts.

Our risk tolerance measure makes available many parallel and intuitive results for the economy as a whole on time preference, precautionary savings motive, and the response of aggregate savings to aggregate shocks. A simple decomposition identity illuminates the way. Market-revealed (aggregate) risk aversion is a weighted average of the individual risk aversion in risk tolerance measure, implying that its response to shocks is merely the average of individuals' responses plus the response of the risk tolerance measure itself to such movements. This latter term arises from the equilibrium risk sharing among agents, and is responsible for many noteworthy effects in the 
aggregation dynamics presented below. If, as is usually assumed, there is a long-term upward drift in endowments, risk tolerance, despite bouncing around with output shocks, will drift upwards as well.

Like risk aversion, the market-revealed time discount factor is the weighted average value of individual counterparts in the risk tolerance measure. As time rolls forward, more patient agents - who have smaller discount factors - are more willing to defer consumption. Assets shift to their hands, which drives down the aggregate discount rate. This phenomenon exerts downward pressure over time on market-revealed time preference in the economy. Of course, the interaction with aggregate shocks and risk preference can amplify or dampen the pressure 1 Our decomposition identity yields simple expressions for how the discount rate moves with time and supply shocks.

Our story is a story of risk sharing and wealth re-distribution as uncertainties resolve and time passes. Surprisingly, these shifts allow market-revealed characteristics for the equivalent agent to lie outside the range of values held by the agents in the economy. That is, if one were to posit that the observed outcome came from a population of homogeneous agents, the hypothetical representative agent could have values for his preferences or actions that lay beyond those for any agent in the true economy of heterogeneous agents.

Precautionary savings illustrate. The equivalent agent may have stronger savings motive than would even the most prudent actual agent in the heterogeneous world. The explanation is straightforward. Agents facing stochastic output save for a rainy day. A world of heterogeneous agents injects an additional layer of dynamic uncertainty in the economy, since the standings of individuals in the economy change stochastically. This additional dynamic behaves as if it raises the demand for precautionary savings. Thus, we point out that, in heterogeneous-agent economies, the large market-revealed precautionary savings motive is not necessarily associated with the dominance of the precautious agents. Rather, the savings motive is high when risk-sharing dynamic between agents is important, i.e., when agents are sufficiently different in their beliefs, or in risk and time preferences. To illustrate, the risk sharing can push up market-revealed precautionary savings motive even when the mean value of risk aversion in the economy drops. It is well known that precautionary savings powerfully push up bond values and lower interest rates. Then it is possible and natural that the interest rate moves in the same direction with the economy's average risk

\footnotetext{
${ }^{1}$ If more patient agents are more (less) risk tolerant, positive shocks will amplify (dampen) the pressure, and vice versa for negative shocks.
} 
aversion when agents differ in their characteristics.

In a heterogeneous and temperate 2 world, savings and savings motives are also highly sensitive to endowment fluctuations: they increase when economic prospects dim and endowments shrink. This phenomenon is consistent with the observed extraordinarily low real interest rates observed in most developed economies in the period following the 2008 meltdown. Aggressive monetary policy surely contributed, but savings had also skyrocketed due to precautionary concerns. Another remarkable implication is that when interest rates are low, they tend to be unstable in the current general additive utilities setting. This is precisely because, as discussed above, the large savings motives responsible for low interest rate is induced by substantial level of risk sharing and hence is highly sensitive to economic fluctuations. In other words, large savings imply large savings cyclicalities in the models. We establish an analytical and almost universal lower bounds for interest rate volatilities. Within the additive utility framework, our investigation thus uncovers, both qualitatively and quantitatively, the insightful role of savings cyclicality in the long-standing risk-free rate and equity premium puzzles of macroeconomics and finance. In retrospect, it also explains why promising models addressing these puzzles in the literature need to adopt either features beyond additive utility (e.g., habit formation, recursivity) or richer time-series properties for aggregate supply and consumption.

Furthermore, our results on the dynamics of risk aversion and precautionary savings, and their consequences for the movement of savings with the economy, have significant implications for determining the direction and magnitude of volatilities in stock returns. The underlying logic is clear: saving decisions reflect portfolio choices, which are intimately related to the volatility of all asset prices, which in turn are influenced by the sloshing of assets among different classes of agents. This savings dynamic (more specifically, the savings sensitivity to economic fluctuations) plays no role in simple and popular models of the economy that employ a representative agent or two classes of agents holding power utility functions. The critical role of the cyclicality of savings gets obscured in such models. In our models, with a plethora of heterogeneous agents, the cyclicality of savings stands out for its influential role quite beyond risk aversion and precautionary savings. The extent of heterogeneity, i.e., how greatly agents differ, turns out to be critical.

In any market-exchange economy, prices are determined by both the growth rate and volatil-

\footnotetext{
${ }^{2}$ Temperance is a determinant of portfolio choices. It is proportional to the fourth derivative of the utility function. We will characterize this behavior under uncertainty more precisely in a later section.
} 
ity of output (endowments in our models), and by the participants' tastes for risk and tradeoff across time, as well as their beliefs. As far as consumption and risk sharing are concerned, our formulation identifies a simple tradeoff between these two key, but seemingly quite different factors. That is because an interesting duality emerges. An economy whose agents differ on time and risk preferences is isomorphic to another economy whose agents differ merely on risk aversion, though the evolution of the endowment in the second economy will differ from what it is in the first. The isomorphism means that consumption partitions, risk sharing between agents, and market-revealed characteristics are identical in the two economies.

This isomorphism potentially enhances our ability to study economies where agents differ on multiple dimensions. First, the seemingly complex dynamic interactions of market participants in an economy with heterogeneous agents are reduced to those of simpler economy but with a modified output process. In particular, there proves to be an intimate connection between this heterogeneity reduction and the market's "natural" selection (that is, the survival) of agents in the economy. Second, employing this isomorphism may immediately pin down the direction in which additional classes of heterogeneity or expanded heterogeneity (e.g., a mean preserving spread) within an existing class will affect the volatility of asset returns. If the modified volatility of the isomorphic economy's output is lower than that of the original economy, that implies that the expansion in heterogeneity in the original economy tend to shrink the volatility in asset returns. This is simply because the volatility of asset prices increases with output volatility in the first order. The powerful implication of this result is that should endowments change, our bounds on asset return volatilities can be immediately adapted from a world where there are mere differences in risk aversion to one where differences in time preference pile atop those. Our later analysis also allows individuals to differ in their beliefs on how endowments will evolve, what might be thought of as their levels of optimism. Moreover, the isomorphism extends. That is, we can add differences in beliefs to those of time preference and risk aversion, and still find another equivalent economy whose agents differ merely in risk aversion. In other words, the disparities in time preference and optimism can be rotated away by a transformation in the evolution of the output process. Market-revealed characteristics toward risk taking and savings will be identical in the two economies.

The paper is structured as follows. Section 2.1 reports briefly the empirical statistical moments (means and volatilities) of interest rates and equity market returns, which have been extensively 
documented in literature. We also discuss recent estimates of distributions of risk aversion and time preference in the population. Not surprisingly, these show substantial degrees of heterogeneity among individuals. Section 2.2 positions our work and findings with respect to the related literature. Section 3 derives various equivalent forms of the risk tolerance measure and discusses their merits in the aggregation analysis of the economy with heterogeneous agents. Section 4 analyzes the effect of savings behaviors on interest rate volatility and identifies substantial lower bounds given the premise of large savings. Section 5 carries out similar analysis on equity return volatilities and derives a sufficient condition for excess equity return volatilities, as long observed in data. Section 6 shows and analyzes the equivalence between the effect of heterogeneities in time preferences and beliefs, and an appropriate modification in the output statistics. Section 7 concludes. All proofs and derivations are given in the appendices.

\section{Empirical facts and related literature}

This section provides factual material to motivate our study of the linkage between risk sharing and equilibrium asset prices given heterogeneous preferences. First, we recount the observed behaviors of returns on key asset (risk-free bond and stocks). Next, we provide recent evidence from literature surveys showing sizable heterogeneity of market participants' preferences. Models employing homogeneous agents do not capture the richness of the world in which we live. Finally, we discuss the literature most relevant to the current work.

\subsection{Estimates of asset returns' moments and preferences}

Returns on equities and risk-free assets are among the most documented quantities in the empirical finance literature. The behaviors of these returns expose stylized facts that can be "puzzling" from the consumption-based asset pricing perspective.

\section{Risk-free rate and return on equity}

Table 1 reports the recent historical means and standard deviations of aggregate consumption growth, returns on equity and short-term risk-free assets (bills), for Japan, UK and US. All returns are real and in annualized percentage values. For further illustration, table 2 also reports long 
Table 1: Consumption growth, and real return on equity and short-term risk-free debt (annual \%): recent history

\begin{tabular}{|c|c|c|c|c|}
\hline Quantities & $a$ & $\begin{array}{c}\text { Japan } \\
(1970.2-1999.1)\end{array}$ & $\begin{array}{c}\text { UK } \\
(1970.1-1999.2)\end{array}$ & $\begin{array}{c}\text { US } \\
(1970.1-1998.4)\end{array}$ \\
\hline \multirow{2}{*}{$\begin{array}{c}\text { consumption } \\
\text { growth }\end{array}$} & mean & 3.20 & 2.20 & 1.81 \\
\hline & stddev & 2.56 & 2.51 & 0.91 \\
\hline \multirow{2}{*}{$\begin{array}{c}\text { real return } \\
\text { on equity }\end{array}$} & mean & 4.72 & 8.16 & 6.93 \\
\hline & stddev & 21.91 & 21.19 & 17.56 \\
\hline \multirow{2}{*}{$\begin{array}{c}\text { real return } \\
\text { on bills }\end{array}$} & mean & 1.39 & 1.30 & 1.49 \\
\hline & stddev & 2.30 & 2.96 & 1.69 \\
\hline \multicolumn{2}{|l|}{$\begin{array}{c}\text { Equity } \\
\text { premium }\end{array}$} & 3.33 & 6.86 & 5.44 \\
\hline
\end{tabular}

${ }^{a}$ Source: Campbell (2003)

Table 2: Equity premia (annual \%): long history

\begin{tabular}{|c|c|c|c|c|}
\hline \hline \multicolumn{2}{|c|}{} & $\begin{array}{c}\text { Japan } \\
(1900-2005)\end{array}$ & $\begin{array}{c}\text { UK } \\
(1900-2005)\end{array}$ & $\begin{array}{c}\text { US } \\
(1900-2005)\end{array}$ \\
\hline \hline \multirow{2}{*}{$\begin{array}{c}\text { Equity } \\
\text { premium }\end{array}$} & mean & 9.84 & 6.14 & 7.41 \\
\cline { 2 - 5 } & stddev & 27.82 & 19.84 & 19.64 \\
\hline
\end{tabular}

${ }^{a}$ Sources: Dimson, Marsh and Staunton (2008)

historical equity risk premia for these countries. In all three countries, for both recent and long histories, real risk-free rates are both low and stable, compared to much higher and more volatile returns on equities. This is the risk-free rate puzzle (Weil (1989)). Similarly, equity premia are also large and volatile vis-a-vis low and stable aggregate consumption growth ${ }^{3}$ This is the closely related equity premium puzzle (Mehra and Prescott (1985)).

\section{Heterogeneity in risk and time preferences}

Our analysis includes heterogeneity in both risk and time preferences, thus it is important to determine whether there is heterogeneity in such dimensions in the real world. Table 3 reports the results of some recent studies on the distribution of individuals' relative risk aversion, $R=$ $\frac{-c \partial^{2} U / \partial c^{2}}{\partial U / \partial c}$, which have been conducted on the US and Norway populations. The first three estimates are obtained from responses to different surveys, over different periods. The surveys employed various forms of hypothetical gambles. The last estimate is inferred from actual financial decisions

\footnotetext{
${ }^{3}$ Dividend growths are also much less volatile than returns on equities.
} 
Table 3: Heterogeneity in Individuals' relative risk aversion $R$

\begin{tabular}{|cc|cc|}
\hline \hline Country & Method & $\begin{array}{c}\text { RRA } \\
R\end{array}$ & $\begin{array}{c}\text { Standard } \\
\text { deviation }\end{array}$ \\
\hline \hline & & & \\
$\mathrm{US}^{a}$ & Surveys & 12.07 & 16.58 \\
$\mathrm{US}^{\circ}$ & Surveys & 8.2 & 6.8 \\
Norway $^{c}$ & Surveys & 3.92 & 2.94 \\
$\mathrm{US}^{d}$ & Actual financial decisions & 2.85 & 3.62 \\
& & & \\
\hline
\end{tabular}

${ }^{a}$ Sources: Barsky, Juster, Kimball and Shapiro (1997)

${ }^{b}$ Kimball, Sahm and Shapiro (2008)

${ }^{c}$ Aarbu and Schroyen (2009)

${ }^{d}$ Paravisini, Rappoport and Ravina (2010)

of investors in an online person-to-person lending platform. Readers should consult the original sources for details. Clearly, all four studies show substantial heterogeneity in the level of relative risk aversion reported by either survey respondents or actual investors. Table 4 reports estimates

Table 4: Heterogeneity in individuals' time discount rate $\delta$ (annual \%): Estimates from surveys

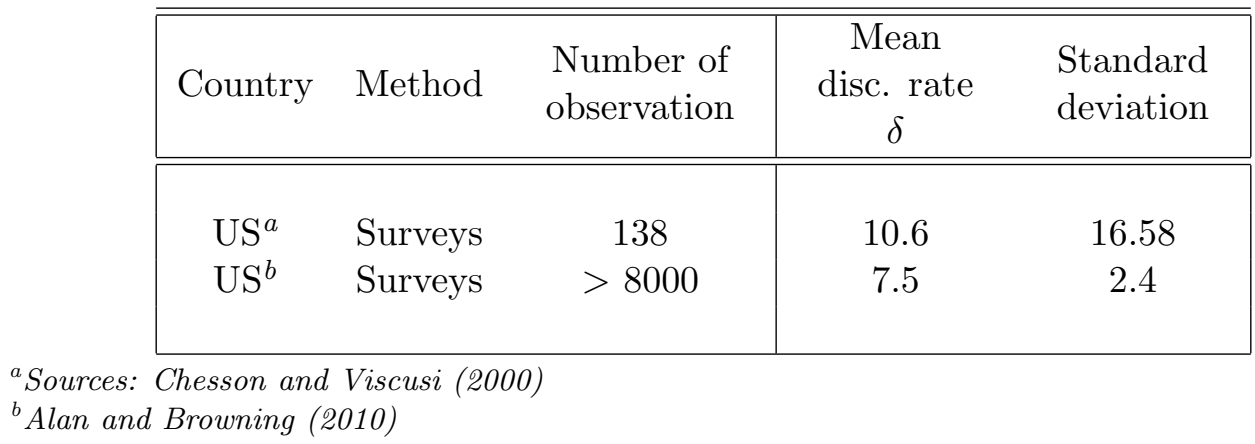

for the distribution of individuals' discount factor $\delta=-\frac{1}{U} \frac{\partial U}{\partial t}$ Both studies found differences in time preference reported by the respondents.

The sizable dispersions in preferences found in these studies motivate our current study of the impacts of heterogeneity on equilibrium asset prices.

\section{$2.2 \quad$ Related literature}

Our paper is most closely related to heterogeneous-agent equilibrium models addressing price anomalies in financial economics literature. The interest on price puzzles has skyrocketed since the seminal papers by Mehra and Prescott (1985) and Weil (1989). Mehra and Prescott (2008)'s 
dedicated handbook offers the most extensive single source of up-to-date references on this important and vibrant topic. The current paper does not attempt to provide new solutions; it instead contributes to a deeper understanding about the nature of risk-free rate and equity premium behaviors within the classic additive utility setting, a setting in which these phenomena are most puzzling. First and conceptually, we shed new light on the crucial role of the cyclicality of precautionary savings in shaping equity and bond return dynamics. Second and analytically, we identify substantial lower bounds on interest rate volatility when interest rates are desirably low. Together, these demonstrate the hard-to-reconcile nature of low and smooth interest rates observed in realworld economies.

In the finance literature, the heterogeneous-agent formulation appeared early on in Benninga and Mayshar (2000), Dumas (1989), Wang (1996) and others, where agents differ in their risk aversions. Heterogeneity in market participants' characteristics has evolved into an attractive topic of active research, which now also incorporates differences in time preferences (Gollier and Zeckhauser (2005), Jouini and Napp (2007), Lengwiler (2005)), beliefs in the fundamentals (Basak (2005), Detemple and Murthy (1994)), or all of the above (Bhamra and Uppal (2010), Lengwiler et al. (2005), Sandroni (2000), Yan (2008)). Heterogeneity generates non-trivial risk sharing patterns and consequently, has rich implications for price dynamics (Bhamra and Uppal (2009), Dumas et al. (2009), Chan and Kogan (2005), Zapatero (1998)), portfolio choices and trading (Gallmeyer and Hollifield (2008), Longstaff and Wang (2008)), and market selection (Blume and Easley (2006), Kogan et al. (2006) and (2009)). In contrast with these works, our paper points out an intuitive tradeoff between agent-based heterogeneities and macroeconomic conditions, which is helpful in analyzing agents' equilibrium interaction and the resulting price dynamics mentioned above.

The degree of heterogeneity in the economy is plausibly the key determinant of the magnitude of heterogeneity's impact. In particular, Chen, Joslin and Tran (2010) study the impact of heterogeneous beliefs in the likelihood and severity of rare events (e.g., crises, disasters and alike) on asset prices. They point out that the risk premium in the economy may drop even when the average level of pessimism among agents surges. This is because there, the driving force is the dynamic dispersion of beliefs and the associated risk sharing, but not just the mean value of the belief distribution. By showing that subject to sufficient heterogeneity in risk aversion in the economy, the equilibrium interest rate may even increase when the average level of precautionary savings 
motives among agents surges, the current study complements their results in identifying another setting where the risk sharing induced by heterogeneity yields spectacular effects.

\section{Risk tolerance measure and aggregation}

In any economy, be it one of homogeneous or heterogeneous agents, risk taking and savings are determined by the behavior of individual agents. In a heterogeneous world, the dynamic competitive interactions among such agents play a major role in determining aggregate outcomes. To address the interactions that are determined by risk taking propensities, and the ultimate consequences for various aggregates, the concept of risk tolerance proves to be both extremely powerful and convenient. It precisely measures how agents' consumptions move with changes in the aggregate endowment. This section uses risk tolerance measures to derive key market-revealed quantities, including risk aversion, time preference and precautionary savings. The approach neatly separate the contributions of agents' characteristics from their interactions. Many interesting aggregate behaviors of the economy, some known others new, then can be readily elucidated.

\subsection{The setting}

To develop intuitive results on aggregation, we first investigate a general endowment economy with many classes of agents. Within each class, agents have identical preferences 4 but across classes agent risk aversions and time preferences differ. Throughout the paper, the superscript $i$ denotes quantities associated with agent $i$. Agents maximize their general time-separable utilities, which are increasing, concave and three-time continuously differentiable. Agent $i$ 's relative risk aversion (RRA) $R^{i}\left(t, c^{i}\right)$ and subjective discount factor $\delta^{i}\left(t, c^{i}\right)$ generally can be functions of consumption $c^{i}$ and time $t$. Alternatively, we will also study the canonical settings with power utilities to make precise the model's key results. For that case, agents' RRAs are constant and simply denoted $\gamma^{i}$, instead of $R^{i}\left(t, c^{i}\right)$ reserved for more general (non-CRRA) settings. At the outset, each agent $i$ is endowed with a fraction $\theta_{S}^{i}(0)$ of a risky stock paying a stochastic dividend stream $w(t)$. The

\footnotetext{
${ }^{4}$ For this reason, to simplify notation, hereafter we simply use agent (being representative of her own homogeneous class) in place of class (of identical agents).
} 
dividend, which reflects the state of the economy, follows a geometric Brownian process (GBM)

$$
\frac{d w(t)}{w(t)}=\mu^{w} d t+\sigma^{w} d Z(t) \Rightarrow w(t)=w(0) e^{\left(\mu^{w}-\left(\sigma^{w}\right)^{2} / 2\right) t} e^{\sigma^{w} Z(t)}
$$

When $\left(\mu^{w}-\left(\sigma^{w}\right)^{2} / 2\right)>0$ the economy is growing in the long term $\left(\lim _{t \rightarrow \infty} E_{0}[w(t) / w(0)] \rightarrow\right.$ $\infty$ a.s.). A single share of the risky stock is available in the economy for agents to trade. In addition, there is a zero net supply of a riskless asset (money market account, also loosely referred to as bond below) created by the agents. Agents trade these two assets and choose consumption levels to maximize their expected utilities subject to a budget constraint5 and market clearing

$$
\begin{aligned}
& \max _{\left\{c^{i}, \theta^{i}\right\}} E_{0} \int_{0}^{T} u^{i}\left(c^{i}(t), t\right) d t \\
& \text { s.t. } c^{i}(t) d t=\theta_{S}^{i}(t)[w(t) d t+d S(t)]+\theta_{B}^{i}(t) B(t) r(t) d t-d w^{i}(t), \\
& \text { and } \sum_{i} \theta_{S}^{i}(t)=1 ; \quad \sum_{i} \theta_{B}^{i}(t)=0 \quad \forall t
\end{aligned}
$$

where $S(t), B(t)=\exp \left(\int_{0}^{t} r d t\right)$ and $w^{i}=\theta_{B}^{i}(t) B(t)+\theta_{S}^{i}(t) S(t)$ respectively denote stock price, bond price and wealth processes ${ }^{6}$ Since the market is complete, there exists a set of positive constant utility weights $\left\{\lambda^{i}\right\}$ such that the above optimal individual consumption plans also solve the equivalent-agent optimization (see Negishi (1960))

$$
V^{\lambda}(\{w\}) \equiv \max _{\left\{c^{i}\right\}} E_{0} \sum_{i} \frac{1}{\lambda^{i}} \int_{0}^{T} u^{i}\left(c^{i}(t), t\right) d t \quad \text { s.t. } \quad \sum_{i} c^{i}(t)=w(t) \forall t .
$$

As the aggregate constraint holds at all time and states, the optimization problem (3) can be equivalently cast in a static formulation at each time and state (Karatzas et. al. (1987), Cox and Huang (1989))

$$
v^{\lambda}(w(t), t) \equiv \max _{\left\{c^{i}\right\}} \sum_{i} \frac{1}{\lambda^{i}} u^{i}\left(c^{i}(t), t\right) \quad \text { s.t. } \quad \sum_{i} c^{i}(t)=w(t) .
$$

\footnotetext{
${ }^{5}$ Aggregating the budget constraint (2) over all agents we obtain $\sum_{i} d w^{i}(t)=d S(t)$, i.e., the total change in agents' wealths equals the change in value of the single share of stock, which is the net asset of the economy.

${ }^{6}$ Given the infinite time horizon $T \rightarrow \infty$, Lengwiler, Malamud and Trubowitz (2005) shows that this economy's necessary and sufficient condition for equilibrium existence is precisely the boundedness of every agent's expected utility of aggregate endowment

$$
E_{0}\left[\int_{0}^{\infty} u^{i}(w(t), t) d t\right]<\infty \quad \forall i .
$$
}

Note that this condition also assures that the stock price is finite. 
Combining the first order equations with the envelope theorem we obtain the following system of equations satisfied by optimal consumption plans

$$
\frac{1}{\lambda^{i}} u_{c}^{i}\left(c^{i}(t), t\right)=v_{w}(w(t), t) \quad \forall i
$$

Throughout the paper, subscripts denote partial derivatives. Thus, $f_{x}(x, y) \equiv \frac{\partial f(x, y)}{\partial x}$.

\subsection{Risk tolerance measure}

In the economics of uncertainty, the ways agents optimally allocate their consumptions across states and time are determined respectively by their relative risk aversion (RRA) and pure time preference (a.k.a. subjective discount factor). It is convenient to adopt these standard characteristics for an equivalent agent of the aggregate economy. Given a complete market, these characteristics are revealed unambiguously from observed prices, and are attributed to this equivalent agent as if there were only one class of agents in the economy. For this reason, hereafter $R, \delta$ and $T$ are respectively referred to as risk aversion, discount factor and risk tolerance of the market-revealed equivalent agent (hereafter, equivalent agent).

$$
\begin{gathered}
R^{i}\left(c^{i}, t\right) \equiv \frac{-c^{i} u_{c c}^{i}\left(c^{i}, t\right)}{u_{c}^{i}\left(c^{i}, t\right)} \longleftrightarrow R(w, t) \equiv \frac{-w v_{w w}(w, t)}{v_{w}(w, t)}, \\
\delta^{i}\left(c^{i}, t\right) \equiv \frac{-u_{c t}^{i}\left(c^{i}, t\right)}{u_{c}^{i}\left(c^{i}, t\right)} \longleftrightarrow \delta(w, t) \equiv \frac{-v_{w t}(w, t)}{v_{w}(w, t)} \\
T^{i}\left(c^{i}, t\right) \equiv \frac{-u_{c}^{i}\left(c^{i}, t\right)}{u_{c c}^{i}\left(c^{i}, t\right)} \longleftrightarrow T(w, t) \equiv \frac{-v_{w}(w, t)}{v_{w w}(w, t)}
\end{gathered}
$$

The apparent analogy of these market-revealed characteristics with those of single-agent economy aims to capture the whole economy's attitudes, such as discount factor $\delta$, risk aversion $R$ and utility function $v(w)$, as of a single equivalent (representative) agent's. In particular, in the aggregate the above definitions imply $T=\frac{w}{R}$, a relation that also holds at individual level.

Following Wilson (1968), there exists a first very simple aggregation relation on risk tolerance (see also proposition 1 )

$$
T(w, t)=\sum^{i} T^{i}\left(c^{i}, t\right)
$$


which motivates the choice of the risk tolerance measure $\left\{p^{i}\right\}$ as micro-economic building blocks of all these market-revealed characteristics

$$
p^{i}\left(c^{i}(w, t), t\right) \equiv \frac{T^{i}\left(c^{i}, t\right)}{T(w, t)}=\frac{T^{i}\left(c^{i}, t\right)}{\sum^{i} T^{i}(w, t)} \Rightarrow \sum_{i} p^{i}=1 .
$$

This implied normalization together with $p^{i} \in[0,1]$, which holds when all agents are risk averse $\left(T^{i}>0 \forall i\right)$, qualify $\left\{p^{i}\right\}$ as a standard measure.

This measure is formulated to precisely capture a key concept that risk tolerant agents play predominant role in consumption and wealth distribution dynamics. To see this point, we note the following very interesting and intuitive relation

$$
p^{i}\left(c^{i}(w, t), t\right)=c_{w}^{i}(w, t)
$$

This identity shows that risk tolerance measure exactly characterizes the individual optimal consumption responses to an aggregate endowment shock. In equilibrium, more risk-tolerant (i.e., larger $\frac{T^{i}}{T}$ ) agents embrace relatively less smooth consumption paths (i.e., larger $c_{w}^{i}$ ), and necessarily contribute more to economy's reactions to output fluctuations. In comparison, we note that neither the least risk averse agent $\left(\min \left\{R^{i}\right\}\right)$ nor the one who consumes $\operatorname{most}\left(\max \left\{\frac{c^{i}}{w}\right\}\right)$ invariably put up strongest response to the aggregate shocks. This signifies the unique role of risk tolerance measure in determining the risk sharing and consumption partition among agents. As agents save and trade accordingly to realize their optimal consumption plan, asset prices and their volatilities necessarily are contingent on this measure. Establishing this link more quantitatively is a central theme of our subsequent analysis.

Being functions of equilibrium consumptions, $\left\{p^{i}\left(c^{i}, t\right)\right\}$ entirely capture both aggregate fluctuation effects and the dynamics of the competitive interaction between agents. The mere fact that $p^{i} \geq 0 \forall i$ (when all agent are risk averse) immediately implies a known and important result that no agents cut their optimal consumption when the aggregate endowment increases, $d w>0$. Furthermore, agents whose optimal consumptions respond most strongly to an aggregate endowment shock will dominate in this measure. 7 as the following concise result implies.

\footnotetext{
${ }^{7}$ The most widely-used heterogeneity measure in literature is consumption share $\left\{\frac{c^{i}(w, t)}{w(t)}\right\}$, which is less expressive with respect to the rich dynamics of equilibrium consumption's changes under supply shocks.
} 
Proposition 1 The equivalent RRA, discount factor and risk tolerance of the entire economy are related to their single-agent counterparts as follows

$$
\begin{aligned}
R(w, t) & =\sum_{i} \frac{T^{i}\left(c^{i}, t\right)}{T(w, t)} R^{i}\left(c^{i}, t\right)=E_{\left\{p^{i}\right\}}\left[R^{i}\right], \\
\delta(w, t) & =\sum_{i} \frac{T^{i}\left(c^{i}, t\right)}{T(w, t)} \delta^{i}\left(c^{i}, t\right)=E_{\left\{p^{i}\right\}}\left[\delta^{i}\right], \\
T(w, t) & =\sum_{i} T^{i}\left(c^{i}, t\right),
\end{aligned}
$$

where $E_{\left\{p^{i}\right\}}[\ldots]$ denotes the expectation under risk tolerance measure $\left\{p^{i}=\frac{T^{i}}{T}\right\}$. This result generalizes the time preference aggregation obtained in Gollier and Zeckhauser (2005) to stochastic settings. (See also Lengwiler, Malamud and Trubowitz (2005) for a discrete-time formulation of the results). Both market-revealed RRA and discount factor are expressed succinctly as averages in risk tolerance measure 8 These representations elucidate many important properties of this economy. Indeed, (8) indicates $R, T>0$, and then $v_{w}>0, v_{w w}<0$ respectively by virtue of eqs. (5), (6), guaranteeing the desired risk-averse and increasing utility for the equivalent agent.

In the stochastic and complete market, agents perfectly share their risks by taking stochastic positions in both stock and bonds. The optimal consumption plans thus are necessarily stochastic, and so are their risk tolerance measures (also referred to as weights), $p^{i}=c_{w}^{i}$. The resulting equivalent preference characteristics e.g., $R, \delta$, are stochastic, not necessarily because their agentbased counterparts e.g., $R^{i}, \delta^{i}$ are stochastic, but rather because their dynamics weights $\left\{p^{i}\right\}$ bounce stochastically. Indeed, in a CRRA utilities setting, the individual $R^{i}, \delta^{i}$ are constant, yet $R, \delta$ in (8) are not so, obviously. To understand those dynamics more precisely, it is best to see how the risk tolerance measure changes under aggregate supply shocks

$$
\frac{d p^{i}(w, t)}{d w}=c_{w w}^{i}(w, t)=\frac{T^{i}\left(c^{i}, t\right)}{(T(w, t))^{2}}\left(T_{c}^{i}\left(c^{i}, t\right)-T_{w}(w, t)\right)
$$

which simplifies in the CRRA utilities setting to

$$
c_{w w}^{i}=\frac{T^{i}\left(c^{i}, t\right)}{(T(w, t))^{2}}\left(\frac{1}{\gamma^{i}}-E_{\left\{p^{i}\right\}}\left[\frac{1}{\gamma^{i}}\right]\right) ; \quad \frac{c_{w w}^{i}}{c_{w}^{i}}=\frac{1}{T(w, t)}\left(\frac{1}{\gamma^{i}}-E_{\left\{p^{i}\right\}}\left[\frac{1}{\gamma^{i}}\right]\right) .
$$

\footnotetext{
${ }^{8}$ That is, weighted averages, with weights being the risk tolerance measures $\frac{T^{i}}{T}$.
} 
These imply that the least risk averse agent $i\left(\gamma^{i}=\gamma^{\text {min }}\right)$ has convex consumption $c_{w w}^{i}>0$ and her weight $c_{w}^{i}$ unambiguously increases with aggregate endowment. The converse holds for the most risk averse agent $\left(\gamma^{\max }\right)$. In between, the transition is monotonic: percentage changes in less risk averse agents' weights $c_{w}^{i}$ are more dramatic than those of more risk averse ones. The stochastic nature of risk tolerance measures is induced by risk sharing mechanism and has profound implications for the volatilities of all market-revealed characteristics, as the latter are some form of weighted averages in this measure. This observation is reflected in the following result, which provides the basis for many findings presented below.

Proposition 2 Suppose $\left\{a^{i}\right\}$ are some agent-based characteristics. The response of the resulting risk-tolerance aggregate $E_{\left\{p^{i}\right\}}\left[a^{i}\right]$ to an aggregate supply shock dw can be decomposed into two components

$$
\frac{\partial E_{\left\{p^{i}\right\}}\left[a^{i}\right]}{\partial w}=E_{\left\{p^{i}\right\}}\left[a_{w}^{i}\right]+\operatorname{Cov}_{\left\{p^{i}\right\}}\left(\frac{p_{w}^{i}}{p^{i}}, a^{i}\right) .
$$

Of special interest, the second component is exclusively associated with the dynamic behavior $p_{w}^{i} \equiv$ $\frac{\partial p^{i}}{\partial w}$ of individual risk tolerance $p^{i}(w, t)$.

To a lesser degree, the first component is also related to risk-tolerance measures, because $a_{w}^{i}=$ $a_{c}^{i} c_{w}^{i}=a_{c}^{i} p^{i}$. But it is primarily associated with the dependence $a^{i}\left(c^{i}, t\right)$ at the agent-specific level at the onset. The mechanism underlying this decomposition is very intuitive. For a simple illustration, let us continue with eq. 10 and assume that all the $a^{i}$ are constant. Dividing both sides of 10 by $p^{i}=T^{i} / T$ yields

$$
\frac{p_{w}^{i}}{p^{i}}=\frac{1}{T(w, t)}\left(\frac{1}{\gamma^{i}}-T_{w}(w, t)\right)
$$

Clearly, $\gamma^{i}<\gamma^{j} \Rightarrow \frac{p_{w}^{i}}{p^{i}}>\frac{p_{w}^{j}}{p^{j}}$, or percentage changes in weights $p^{i}$ are greatest for agents with lesser risk aversion $\gamma^{i}$. This is because under a positive shock $d w>0$ to the aggregate endowment, less risk averse agents, who invest disproportionally in the risky contingent claim on aggregate wealth (stock) become relatively better off, and contribute more to the welfare. Indeed, in this CRRA framework, (11) simplifies to

$$
\frac{\partial E_{\left\{p^{i}\right\}}\left[a^{i}\right]}{\partial w}=\frac{1}{T(w, t)} \operatorname{Cov}_{\left\{p^{i}\right\}}\left(\frac{1}{\gamma^{i}}, a^{i}\right) .
$$


The situations when $a^{i}>a^{j}$ for $\gamma^{i}>\gamma^{j}$ and vice versa are referred to as comonotone. Similarly, anti-comonotonicity means $a^{i}>a^{j}$ if $\gamma^{i}<\gamma^{j}$ and vice versa. To illustrate, when $a^{i}$ is the discount rate $\delta^{i}$, comonotone relations represent the normal case where less risk averse agents also tend to be more patient. We see that when $\left\{a^{i}\right\}$ and $\left\{\gamma^{i}\right\}$ are comonotone, the mean value $E_{\left\{p^{i}\right\}}\left[a^{i}\right]$ decreases unambiguously with aggregate endowment $w$. This is precisely because smaller values of $a^{i}$ (associated with smaller $\gamma^{i}$ by co-monotonicity) have relatively larger weights after a positive shock increases $w$ as we argued above, and thus drive down the mean value. The opposite holds when $\left\{a^{i}\right\}$ and $\left\{\gamma^{i}\right\}$ are anti-comonotone; larger $a^{i}$ (associated with smaller $\gamma^{i}$ by anti-comonotonicity) have relatively larger weights after a positive shock increases $w$, which makes mean value $E_{\left\{p^{i}\right\}}\left[a^{i}\right]$ increases unambiguously with aggregate endowment $w$.

Two immediate applications concern the market-revealed risk aversion $R$ and discount rate $\delta$ of proposition 1, specialized to the CRRA utilities setting 99

$$
\begin{aligned}
R_{w}(w, t) & =\frac{1}{T(w, t)} \operatorname{Cov}_{\left\{p^{i}\right\}}\left(\gamma^{i}, \frac{1}{\gamma^{i}}\right)<0 \\
\delta_{w}(w, t) & =\frac{1}{T} \operatorname{Cov}_{\left\{p^{i}\right\}}\left(\frac{1}{\gamma^{i}}, \delta^{i}\right) .
\end{aligned}
$$

The first equation demonstrates a well-known result of decreasing market-revealed risk aversion (see e.g., Wang (1996)). The second formalizes the wealth effect on market-revealed time preference first obtained in Gollier and Zeckhauser (2005). We recast these known and important results in connection with the risk tolerance measure to capture the key intuitions underlying this measure's dynamics.

The above market-revealed characteristics also yields the equivalent hyperbolic discounting behavior of the economy (Gollier and Zeckhauser (2005)). Taking the derivative with respect to time, $\delta_{t} \equiv \frac{\partial \delta}{\partial t}$, again within the CRRA setting yields

$$
\delta_{t}(w, t)=-\sum_{i} p^{i}(w, t) \frac{\left(\delta(w, t)-\delta^{i}\right)^{2}}{\gamma^{i}}<0 .
$$

The intuition again can be distilled from competitive interaction in equilibrium. More patient agents are more willing to defer their consumptions, and thus will increase their dominance as time

\footnotetext{
${ }^{9}$ Corresponding expression for non CRRA setting is $R_{w}=\frac{1}{T} \operatorname{Cov}_{\left\{p^{i}\right\}}\left(\gamma^{i}, T_{c}^{i}\right)$, see 67).
} 
rolls forward. Given that being more patient means having smaller $\delta^{i}$, this competitive behavior simply decreases the weighted average discount factor $\delta(w, t)$ over time. This in turn has interesting and direct effects on the term structure of interest rates (Lengwiler(2005)).

When heterogeneities are present in both risk and time preference, either a low risk aversion or a small discount rate will lead an individual to play a greater role in the long run We will analyze quantitatively the tradeoff between these characteristics in conjunction with agents' longrun survival in section 6 ,

\subsection{Market-revealed precautionary savings}

Prudence (see Leland (1968) and Sandmo (1970)) is a key characteristic determining precautionary savings, and thus both interest rate and returns on other assets. Kimball (1990) shows that the prudence, defined in analogy with relative risk aversion (6) as

$$
P^{i}\left(c^{i}, t\right) \equiv \frac{-c^{i} u_{c c c}^{i}\left(c^{i}, t\right)}{u_{c c}^{i}\left(c^{i}, t\right)} \longleftrightarrow P(w, t) \equiv \frac{-w v_{w w w}(w, t)}{v_{w w}(w, t)}
$$

provides an analytical measure of the intensity of the precautionary savings motive. Other factors being equal, an agent $i$ who is more prudent (larger $P^{i}$ ) will save relatively more under the prospect of future income uncertainties. For a heterogeneous-agent economy with general additive utilities, we can differentiate the FOC (3) twice to obtain the explicit aggregation relation

$$
P(w, t)=E_{\left\{p^{i}\right\}}\left[P^{i}\left(c^{i}, t\right)\right]-\operatorname{Cov}_{\left\{p^{i}\right\}}\left(R^{i}\left(c^{i}, t\right), \frac{1}{R^{i}\left(c^{i}, t\right)}\right)+\operatorname{Cov}_{\left\{p^{i}\right\}}\left(R^{i}\left(c^{i}, t\right), \frac{c^{i} R_{c}^{i}\left(c^{i}, t\right)}{\left(R^{i}\left(c^{i}, t\right)\right)^{2}}\right),
$$

where the moments again are defined in the risk tolerance measure. The key observation is that while market-revealed risk aversion (8) has value bounded within the spectrum of agents' RRA $\left(R^{\min } \leq R(w, t) \leq R^{\max }\right)$, such bounding need not apply for market-revealed prudence $P(w, t)$. The market-revealed precautionary savings motive contains a weighted average $E_{\left\{p^{i}\right\}}\left[P^{i}\right]$ over individual agents, which plausibly results from a simple aggregation. More profoundly, it also contains additional components which arise from the dynamics of the risk sharing, and thus the risk tolerance measure itself, much in the spirit of the mechanism underlying propososition 2 . To illustrate 
this insight, let us employ the class of power utilities, wherein (15) becomes

$$
P(w, t)=E_{\left\{p^{i}\right\}}\left[P^{i}\left(c^{i}, t\right)\right]-\operatorname{Cov}_{\left\{p^{i}\right\}}\left(\gamma^{i}, \frac{1}{\gamma^{i}}\right)=E_{\left\{p^{i}\right\}}\left[P^{i}\right]-T \sum_{i} \gamma^{i} c_{w w}^{i} .
$$

As $p^{i}=c_{w}^{i}$ defines the risk tolerance measure, $c_{w w}^{i}$ clearly characterizes the dynamics of this measure under changes in aggregate endowment $w$. Individual agents' savings are not made independently as naive intuition about aggregation might suggest. That is because the economy's precautionary savings reflect both agents' average precautionary savings motive and the response to stochastic wealth distribution $\sqrt{10}$ This second factor inflates the market-revealed precautionary savings motive because the term $\operatorname{Cov}_{\left\{p^{i}\right\}}\left(\gamma^{i}, \frac{1}{\gamma^{i}}\right)$ is invariably negative. The more risk averse agents have concave consumptions $\left(c_{w w}^{i}<0\right.$, see $(10)$ ), and they contribute positively to this induced prudence due to their larger $\gamma^{i}$. When agents are sufficiently different in their risk preferences, this covariance tends to be large (and negative) and it can inflate economy's savings motive greatly beyond that of even the most prudent agent in the economy. The proposition 3 and figure 1 below confirm this extraordinary effect stemming from risk sharing between agents.

Before turning to the main results of this section, we note that there exists another relation involving prudence $P(w, t)$, directly obtained from the definitions of $R$ and $P$ (derived in appendix A)

$$
R_{w}(w, t)=\frac{R(w, t)}{w}(1+R(w, t)-P(w, t))
$$

This equality does not rely on any aggregation mechanism, and hence holds at both the agent and aggregate level. (17) implies that high market-revealed precautionary savings are related to the countercyclicality in market-revealed risk aversion. We will discuss this cyclicality and its implication for interest rate volatility in more detail in section 4. Many important properties related to risk sharing between agents emerge in a world with merely two classes of agents. We

\footnotetext{
${ }^{10}$ We may also see this quantitatively in the equivalent agent's optimization problem in a simple two-period model. The equivalent agent optimally chooses current savings $X$ subject to initial wealth constraint $W$ and future uncertain income $\tilde{Y}$

$$
\begin{aligned}
& \max _{X}\left[v(W-X, t)+E_{t} v(X+\tilde{Y}, t+1)\right]= \\
& \max _{X}\left\{\max _{\sum c^{i}(t)=W-X ; \sum c^{i}(t+1)=X+\tilde{Y}} \sum_{i} \frac{1}{\lambda^{i}}\left[u^{i}\left(c^{i}, t\right)+E_{t} u^{i}\left(c^{i}, t+1\right)\right]\right\} .
\end{aligned}
$$

Evidently, equivalent agent's precautionary savings optimization composes of two-stage optimization over agents', subject to market clearings in each period. This subtle constraints constitute additional sensitivity of social utility to future uncertainty that equivalent agent should be wary of.
} 
find it very helpful in various places to present these results in a two-agent economy.

Proposition 3 1. In the multiple-CRRA-agent economy, market-revealed precautionary savings are

$$
P(w, t)=E_{\left\{p^{i}\right\}}\left[P^{i}\left(c^{i}, t\right)\right]-\operatorname{Cov}_{\left\{p^{i}\right\}}\left(\gamma^{i}, \frac{1}{\gamma^{i}}\right),
$$

and thus is always larger than or equal to the average individual precautionary savings $E_{\left\{p^{i}\right\}}\left[P^{i}\left(c^{i}, t\right)\right]$ in risk tolerance measure $\left\{p^{i}\right\}$.

2. The market-revealed precautionary savings in the two-CRRA-agent economy are a concave quadratic function of $p^{A}$

$$
P(w, t)=\left(p^{A}(w, t) \gamma^{A}+p^{B}(w, t) \gamma^{B}\right)\left(1+\frac{p^{A}(w, t)}{\gamma^{A}}+\frac{p^{B}(w, t)}{\gamma^{B}}\right)
$$

When individual $R R A \gamma^{A}, \gamma^{B}$ satisfy $\frac{\gamma^{B}}{\gamma^{B}+1}>\gamma^{A}$, there exists a region of consumption distribution between the two agents where the market-revealed precautionary savings are higher than that of either agent

$$
P^{*}>\max \left\{P^{A}=\gamma^{A}+1 ; P^{B}=\gamma^{B}+1\right\} .
$$

To illustrate the results of proposition 3 , Figure 1 plots the market-revealed prudence in a twoCRRA-agent economy with $\gamma^{A}=0.1$ and $\gamma^{B}=10$. In this case, $P$ is a function of first agent's risk tolerance weight $p^{A} \equiv \frac{c^{A} \gamma^{B}}{c^{A} \gamma^{B}+c^{B} \gamma^{A}}$. Following the pattern of eq. 16 , we decompose this aggregate into two components; the weighted average prudence and the dynamics-induced prudence. We see that the maximum market-revealed prudence $P \sim 30$ is reached at $p^{A}=\frac{c^{A} \gamma^{B}}{c^{A} \gamma^{B}+c^{B} \gamma^{A}} \sim 0.6$ (or $\left.\frac{c^{A}}{c^{B}} \sim 1.5 \%\right)$. This value far exceeds either individual prudence level, $P^{A}=1.1, P^{B}=11$. The excess stems from the risk sharing mechanism, and is quantified by the risk tolerance measure dynamic. The latter tends to zero in both homogeneous limits $\left(p^{A}=0,1\right)$ where the risk sharing possibility between the agents vanishes. Collectively, the agents may keep up this high marketrevealed precautionary savings motive for an extended period of time because they differ as well in time preference 11 We will study in detail how precautionary savings affect both the levels and

\footnotetext{
${ }^{11}$ Yan (2008) shows that no agent dominates the others in the long run when they have similar "survival index" values $\delta^{A}+\gamma^{A}\left(\mu^{w}-\frac{\left(\sigma^{w}\right)^{2}}{2}\right) \approx \delta^{B}+\gamma^{B}\left(\mu^{w}-\frac{\left(\sigma^{w}\right)^{2}}{2}\right)$. For current parameters $\gamma^{A}=0.1, \gamma^{B}=10$, this co-survival
} 


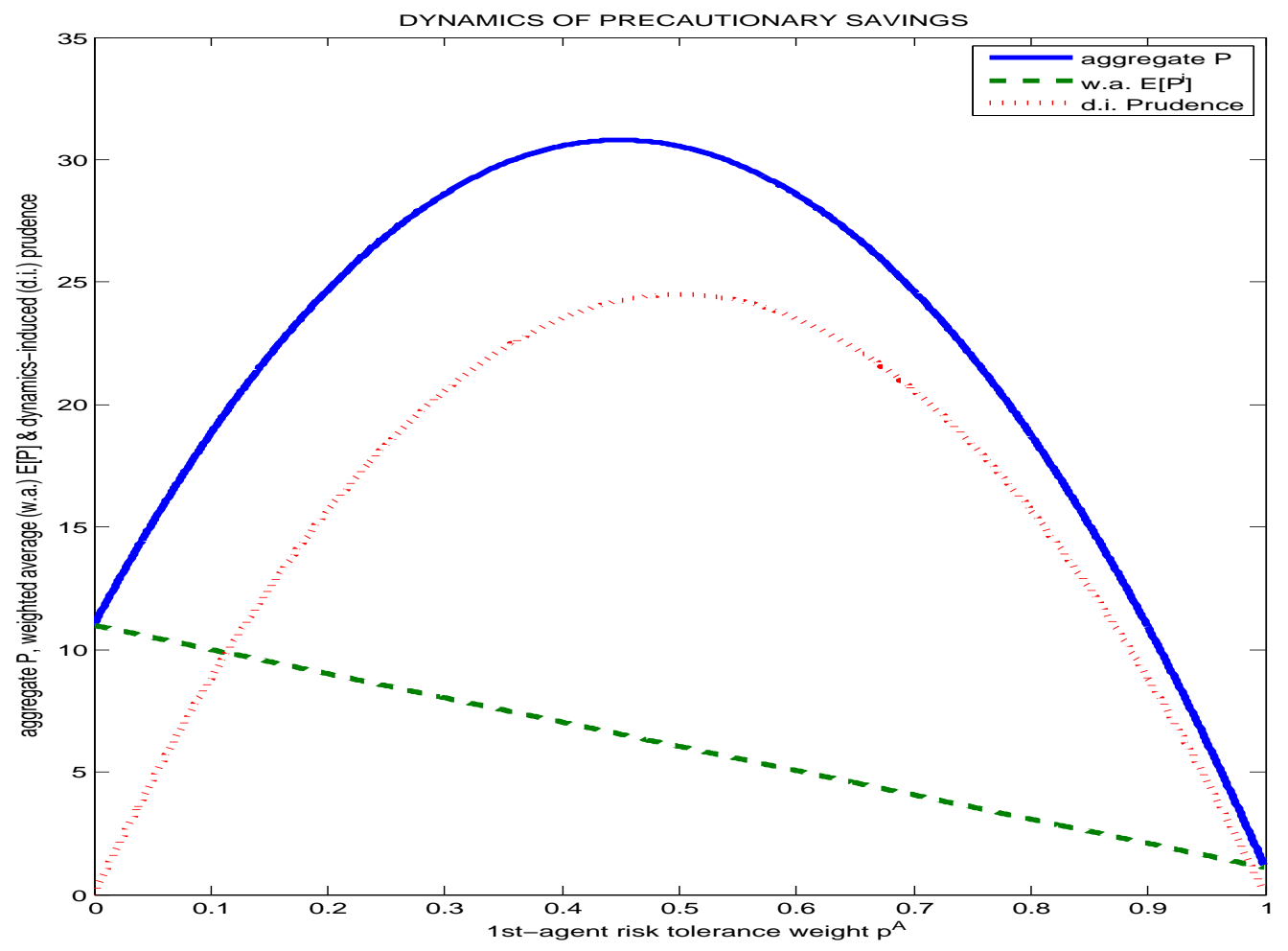

Figure 1: Two-CRRA-agent economy: $\gamma^{A}=0.1, \gamma^{B}=10$. Market-revealed (aggregate) prudence $P\left(p^{A}\right)$ and its components (18): weighted average (w.a.) $E_{\{p\}}\left[P^{i}\right]$ and dynamics-induced (d.i.) prudence $-\operatorname{Cov}_{\left\{p^{i}\right\}}\left(\gamma^{i}, \frac{1}{\gamma^{i}}\right)$. These are plotted against agent $A$ 's risk tolerance weight $p^{A}=\frac{T^{A}}{T^{A}+T^{B}}=\frac{c^{A} \gamma^{B}}{c^{A} \gamma^{B}+c^{B} \gamma^{A}}$.

volatilities of asset returns in later sections.

\subsection{Cyclicality of market-revealed precautionary savings}

We now delve deeper into the microeconomic foundations of asset pricing to see how the cyclicality of precautionary savings motive moves with consumption and wealth. This analysis provides rigorous grounds to study the key effects of savings cyclicality on equilibrium price behaviors in later sections. condition holds, e.g., when subjective discount rates are $e^{-\delta^{A}} \approx 0.8, e^{-\delta^{B}} \approx 1$. 
Central to our analysis is a simple and strong relation between precautionary savings motive $P(w, t)$ and its cyclicality $P_{w}(w, t)$ that holds for any general time separable utility.

$$
P_{w}(w, t)=\frac{P(w, t)}{w}(1+P(w, t)-Q(w, t))
$$

where $Q(w, t)$ is referred to as temperance

$$
Q^{i}\left(c^{i}, t\right) \equiv \frac{-c^{i} u_{c c c c}^{i}\left(c^{i}, t\right)}{u_{c c c}^{i}\left(c^{i}, t\right)} \longleftrightarrow Q(w, t) \equiv \frac{-w v_{w w w w}(w, t)}{v_{w w w}(w, t)}
$$

Kimball $(1992,1993)$ shows that in a partial equilibrium setting with multiple sources of risks, temperance affects the allocation of savings between safe and risky assets, i.e., portfolio choice. First, in light of the relation (20), temperance $Q(w, t)$ contributes decisively to the cyclicality of savings. This savings adjustment in turn is reflected in asset return volatilities ans asset (bond and stock) holdings:12 In the current general equilibrium settings, our observation in 20 thus reinforces Kimball's partial equilibrium results.

Second and more important, equation 20 constitutes a new and keen relation between savings and savings cyclicality in general heterogeneous-agent settings; savings behaviors tend to be more volatile when savings motives are higher! Indeed, all else being equal, the intensity of cyclicality $P_{w}$ increases more than linearly with $P^{13}$ in 20 . This finding is somewhat unexpected since a priori savings and volatility of savings may not necessarily be tightly bound. A counter-example illustrates this point. When the representative agent conventionally has CRRA utility of the form $U(C, t) \sim \frac{C^{1-\gamma}}{1-\gamma}$, the precautionary savings motive $P=\gamma+1$ is constant, and thus savings cyclicality is null, regardless of how big this savings motive $P$ is. In contrast, the intuition behind our observation (20) highlights the risk sharing dynamics in an environment with heterogeneous agents. As we saw in the last section, in such setting the aggregate savings motive $P$ is high not because the most precautious agent dominates the economy. Rather, large $P$ arises when risk sharing dynamics are important, which are possible on the premise that agents sufficiently differ in their characteristics, as illustrated by figure 1. Precisely because of this marked heterogeneity in agents' risk preferences, shocks to the output induce considerable amount of assets and wealth changing

\footnotetext{
${ }^{12}$ Given complete market hedging, portfolio choices are one-to-one with asset return volatilities. One's position in the stock is the ratio of wealth volatility to stock price volatility.

${ }^{13} Q$ may also change with $P$. But in a setting with many agents, this dependence is rather weak.
} 
hands among investors. As a result, economy's savings behavior is then highly sensitive to output fluctuation.

To illustrate, we establish the aggregation relations concerning temperance, along the lines similar to our analysis of market-revealed precautionary savings. For simplicity, we consider again the power utilities setting ${ }^{14}$ Differentiating the FOC (5) repeatedly yields the analytical expression of market-revealed temperance $Q(w, t)$

$$
Q(w, t)=E_{\left\{p^{i}\right\}}\left[Q^{i}\right]-2 \operatorname{Cov}_{\left\{p^{i}\right\}}\left(\gamma^{i}, \frac{1}{\gamma^{i}}\right)-\frac{R^{2}(w, t)}{P(w, t)} \operatorname{Var}_{\left\{p^{i}\right\}}\left(\frac{1}{\gamma^{i}}\right)
$$

Given that market-revealed temperance arises from the third order derivative of the FOC, the dynamics of risk sharing, and thus risk tolerance measure, contribute two terms beyond the naive weighted average of individual temperance. This basic intuition also emerges from proposition 2. In the difference with prudence, for temperance the contribution of risk tolerance measure dynamics is both strong and ambiguous. The market-revealed $Q$ can either be larger than the largest $Q^{i}$, or smaller than the smallest $Q^{i}$. In analogy with proposition 3 , when specializing to the two-CRRA-agent economy, we can specifically assess the market-revealed tolerance $P(w, t)$ and temperance $Q(w, t)$ on a comparative basis. This comparison is important since both direction and quantitative behavior of savings cyclicality $P_{w}(20)$ are determined by the relative importance of $P$ and $Q$.

Proposition 4 The market-revealed temperance in the two-CRRA-agent economy is a simple rationalpolynomial function of first agent's risk tolerance weight $p^{A}$ (note: $p^{B}=1-p^{A}$ )

$$
Q(w, t)=\left(p^{A}(w, t) \gamma^{A}+p^{B}(w, t) \gamma^{B}\right)\left[3\left(\frac{p^{A}(w, t)}{\gamma^{A}}+\frac{p^{B}(w, t)}{\gamma^{B}}\right)+\frac{1-\frac{p^{A}(w, t)}{\left(\gamma^{A}\right)^{2}}-\frac{p^{B}(w, t)}{\left(\gamma^{B}\right)^{2}}}{1+\frac{p^{A}(w, t)}{\gamma^{A}}+\frac{p^{B}(w, t)}{\gamma^{B}}}\right]
$$

which can be either positive or negative. There always exists a consumption region determined by

$$
p^{A}\left(c^{A}, t\right)>\max \left\{0, \frac{1}{2}+\frac{1}{2} \frac{\gamma^{A} \gamma^{B}}{\left(\gamma^{A}-\gamma^{B}\right)}\right\}
$$

within which market-revealed precautionary savings motive is countercyclical; $P_{w}<0$.

\footnotetext{
${ }^{14}$ We derive general results for any additive utilities in the appendix A
} 
As mentioned above, the cyclicality of $P$ should influence interest rate smoothness. Hence this proposition provides an important precursor to assessing the volatilities of asset returns in this economy. Those results will be reported in proposition 5 . To illustrate, Figure 2 plots the marketrevealed temperance $Q\left(p^{A}\right)$ together with its two components: the weighted average temperance (first term of (21)) and the dynamics-induced temperance (last two terms of (21)). Each is a function of the first agent's risk tolerance weight $p^{A}=\frac{T^{A}}{T}=\frac{c^{A} \gamma^{B}}{c^{A} \gamma^{B}+c^{B} \gamma^{A}}$ in the illustrative twoCRRA-agent economy (with $\gamma^{A}=0.1, \gamma^{B}=10$ ). Clearly, unlike market-revealed RRA $R(w, t)$, $Q(w, t)$ is not bounded by individual CRRA temperances $Q^{i}=\gamma^{i}+2$. For a certain range of

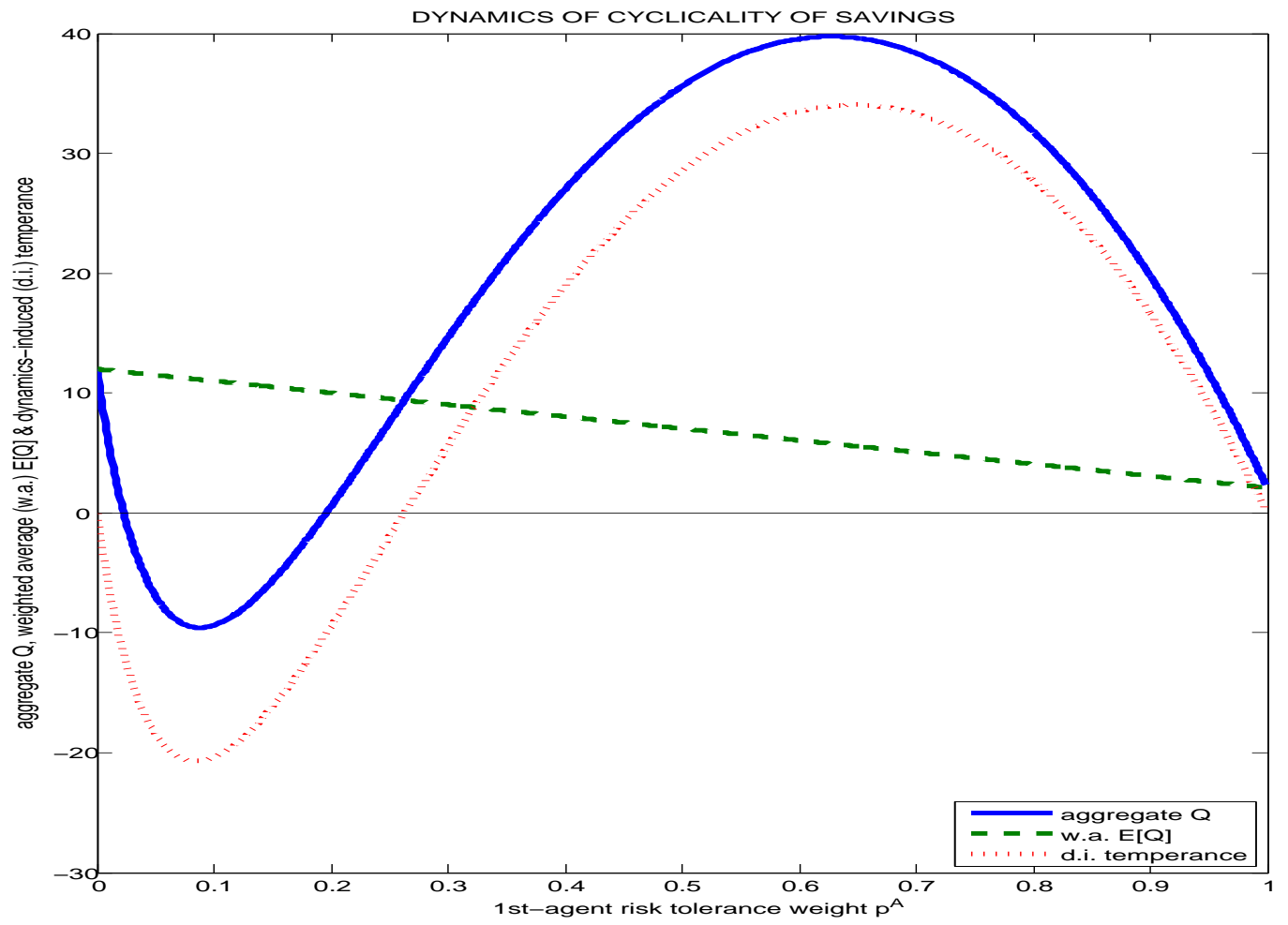

Figure 2: Two-CRRA-agent economy: $\gamma^{A}=0.1, \gamma^{B}=10$. Market-revealed (aggregate) temperance (savings cyclicality) $Q$ and its components $(21)$ : weighted average (w.a.) $E_{\{p\}}\left[Q^{i}\right]$ and dynamics-induced (d.i.) savings cyclicality $-2 \operatorname{Cov}_{\left\{p^{i}\right\}}\left(\gamma^{i}, \frac{1}{\gamma^{i}}\right)-\frac{R^{2}(w, t)}{P(w, t)} \operatorname{Var}_{\left\{p^{i}\right\}}\left(\frac{1}{\gamma^{i}}\right)$. These are plotted against agent $A^{\prime}$ 's risk tolerance weight $p^{A}=\frac{T^{A}}{T^{A}+T^{B}}=\frac{c^{A} \gamma^{B}}{c^{A} \gamma^{B}+c^{B} \gamma^{A}}$. 
consumption partition, the dynamics-induced temperance is so strong that market-revealed $Q(w, t)$ falls negative albeit all individual $Q^{i}$ s are positive. Again in homogeneous limits $\left(p^{A}=0,1\right)$, the sharing dynamics vanish and so does the dynamics-induced temperance.

Interestingly, with three agents or more in the economy, the market-revealed characteristics $R(w, t), P(w, t), Q(w, t)$ are largely independent of each other, allowing more flexibility to estimate the model in accordance with empirical patterns. This shows the rich outcome of genuine heterogeneities, beyond that of the customary but rigid assumption of a CRRA-representative agent in the literature.

\section{Interest rate volatility}

In this model's complete-market intertemporal setting, no-arbitrage is enforced by the unique state price density $M(w, t)$. In the current consumption-based framework, this state price density is the marginal utility (5) of the equivalent agent

$$
M(w, t)=v_{w}(w, t)
$$

The risk-free rate (rfr) $r$ and the market price of risk (mpr) (or Sharpe ratio) $\eta$ are identified with the drift and volatility of the state price density: $\frac{d M(w, t)}{M(w, t)}=-r(w, t) d t-\eta(w, t) d Z(t)$, and thus

$$
\begin{aligned}
& r(w, t)=\delta(w, t)+R(w, t)\left[\mu^{w}-\frac{1}{2}\left(\sigma^{w}\right)^{2} P(w, t)\right] \\
& \eta(w, t)=\frac{w \sigma^{w}}{T(w, t)}=\sigma^{w} R(w, t) .
\end{aligned}
$$

Here $r(w, t)$ is the instantaneous risk-free rate at time $t$. Throughout this paper, for brevity we also refer to it interchangeably as risk-free rate and interest rate. Both rfr and mpr have forms familiar from a single-agent economy, which justifies the use of the associated characteristics $\{R, P, Q\}$ revealed by market prices as if there were a single equivalent agent representing the current heterogeneous-agent economy. In particular, a strong market-revealed precautionary savings effect is needed to drive down the interest rate's magnitude in 24

$$
P(w, t)>\frac{2 \mu^{w}}{\left(\sigma^{w}\right)^{2}} \sim 100
$$


Here the numerical bound is based on the estimates of the aggregate consumption growth moments $\mu^{w} \sim 2 \%, \sigma^{w} \sim 2 \%$ (Table 1). As we see in proposition 3, the risk-sharing dynamic in heterogeneous-agent economy is able to generate a strong savings motive $P$ out of much smaller individual values $P^{i}$, given that agents differ sufficiently in their risk preference. Similarly, for the stock market to be priced by the above state price density $M(w, t), \operatorname{mpr} \eta$ needs to satisfy the Hansen-Jagannathan bound (Hansen and Jagannathan (1991), see also appendix). By virtue of (24), this constraint too has a very familiar expression in the current heterogeneous-agent setting

$$
\sigma^{w} R(w, t)=\eta(w, t) \geq \frac{\mu^{s}(w, t)-r(w, t)}{\sigma_{\mu^{s}-r}}[1-r(w, t)]
$$

where $\mu^{s}$ and $\sigma_{\mu^{s}-r}$ are respectively the stock market expected return and excess return volatility. In the data, typically the stock market excess return $\mu^{s}-r \sim 6 \%$, the excess volatility $\sigma_{\mu^{s}-r} \sim 20 \%$ and the real $\mathrm{rfr} r \sim 2 \%$, which imply a conservative lower bound ${ }^{15}$ on the aggregate risk aversion

$$
R(w, t) \geq \frac{1}{\sigma^{w}} \frac{\mu^{s}(w, t)-r(w, t)}{\sigma_{\mu^{s}-r}}[1-r(w, t)]>15
$$

The large value for risk aversion implied from the excess stock market return is the well-known main thesis of the equity premium puzzle. In the current section, our main focus is to show analytically that this and specially the large precautionary savings bound (25) also have profound impact on the interest rate volatility. Intuitively, as hinted by the stochastic natures of $r(w, t)$ and $\eta(w, t)$ in (24) as well as the presence of aggregate quantities $R, P$ therein, the heterogeneity among agents necessarily affects the volatilities of asset prices in important ways.

To fix the notation, we adopt the interest rate diffusion process $d r(w, t)=\mu^{r}(w, t) d t+\sigma^{r}(w, t) d Z(t)$ where like $r(w, t)$ itself, the $\mu^{r}, \sigma^{r}$ are endogenous in the model. Indeed, in analogy with (40), the volatility $\sigma^{r}$ of the rfr is

$$
\sigma^{r}(w, t)=w \sigma^{w} r_{w}(w, t) \equiv \sigma_{\Gamma}^{r}(w, t)+\sigma_{\Delta}^{r}(w, t),
$$

\footnotetext{
${ }^{15}$ Both bounds on $P \sqrt{25}$ and $R \sqrt{27}$ are most sensitive to the estimated value of consumption growth volatility $\sigma^{w}$. In the US data (Table 1) $\sigma^{w} \sim 1 \%$. Here we adopt $\sigma^{w} \sim 2 \%$ to have very conservative lower values for the aggregate savings motive and risk aversion, while noting that a smaller value of $\sigma^{w}$ will lead to larger $P, R$ and thus an even more volatile rfr than what we point out in this section.
} 
where

$$
\begin{gathered}
\sigma_{\Gamma}^{r}(w, t) \equiv w \sigma^{w}\left(\mu^{w} R_{w}(w, t)-\frac{\left(\sigma^{w}\right)^{2}}{2}\left[R_{w}(w, t) P(w, t)+R(w, t) P_{w}(w, t)\right]\right) \\
\sigma_{\Delta}^{r}(w, t) \equiv w \sigma^{w} \delta_{w}(w, t)
\end{gathered}
$$

are the components of rfr volatility associated primarily with the heterogeneity in risk aversion and time preference, respectively. The expressions for these components are obtained by computing the partial derivative $r_{w}$ from (24). We now analyze the contribution of each type of heterogeneity to rfr volatility.

Judging from the abundance of the derivatives $R_{w}, P_{w}$ in the above expression of $\sigma_{\Gamma}^{r}$, this component of rfr volatility is necessarily characterized by the response of economy's collective risk preference and savings motive to supply shock $d w$. A closer look helps to estimate the magnitude of this volatility. Plugging (17), (20) into 29 yields

$$
\sigma_{\Gamma}^{r}=\sigma^{w} R(w, t)\left[-\mu^{w}(P(w, t)-R(w, t)-1)+\frac{\left(\sigma^{w}\right)^{2}}{2} P(w, t)(Q(w, t)-R(w, t)-2)\right] .
$$

Terms on the right-hand side simply express the sensitivity of aggregate intertemporal consumption smoothing and precautionary savings behaviors to output fluctuations, as they are derived directly from the last two terms of (24). The most remarkable feature here is that both of these sensitivities are substantial under the afore mentioned premise of large savings motives (25) needed for a low real interest rate. Indeed, both terms in $(30)$ are dominated by the large factor $P$, given the realistic values for aggregate consumption moments $\mu^{w}, \sigma^{w} \sim 2 \%$. This observation then offers a simple but very drastic implication for the interest rate of general heterogeneous-agent economies with additive utilities. Namely, in these models, a realistically low interest rate will tend to be excessively volatile. The following proposition quantifies this important observation in analytical terms.

Proposition 5 Assuming sufficiently large precautionary savings motive (25), in a general economy with agents heterogeneous in their time-additive risk preferences, the interest rate volatility is 
almost always $\sqrt{16}$ bounded from below

$$
\left|\sigma^{r}(w, t)\right|>\mu^{w} \sigma^{w} R(w, t)\left|Q(w, t)-\frac{2 \mu^{w}}{\left(\sigma^{w}\right)^{2}}\right|
$$

More specifically,

$$
\begin{array}{ll}
\sigma^{r}(w, t)>\mu^{w} \sigma^{w} R(w, t)\left(Q(w, t)-\frac{2 \mu^{w}}{\left(\sigma^{w}\right)^{2}}\right)>0 & \text { if } \quad Q(w, t)>\frac{2 \mu^{w}}{\left(\sigma^{w}\right)^{2}}+R(w, t) \\
\sigma^{r}(w, t)<\mu^{w} \sigma^{w} R(w, t)\left(Q(w, t)-\frac{2 \mu^{w}}{\left(\sigma^{w}\right)^{2}}\right)<0 & \text { if } \quad Q(w, t)<\frac{2 \mu^{w}}{\left(\sigma^{w}\right)^{2}}
\end{array}
$$

Qualitatively, a key factor determining the volatility of the $\mathrm{rfr}$ is the cyclicality $P_{w}$ of precautionary savings, quantified by market-revealed temperance $Q(w, t)$ in the above expression. This observation identifies a new and interesting factor driving interest volatility, one that is supported by strong intuitions. Here, a critical connection is the relation 20, i.e., large precautionary savings $P$ tend to induce strong savings cyclicality $\left|P_{w}\right|$. In turn, for large $P(25)$, both the intertemporal consumption smoothing and precautionary savings motives are fiercely sensitive to supply uncertainty as in (30), and the resulting interest rate is highly volatile unless these two sensitivities cancel out. Proposition 5 shows that such cancellation holds only within a range of temperance, $Q \in\left(\frac{2 \mu^{w}}{\left(\sigma^{w}\right)^{2}}, \frac{2 \mu^{w}}{\left(\sigma^{w}\right)^{2}}+R(w, t)\right)$. Given the small empirical values for the consumption moments $\mu^{w}, \sigma^{w} \sim 2 \%$, and a non-extreme value of risk aversion $\left(R \ll \frac{2 \mu^{w}}{\left(\sigma^{w}\right)^{2}}\right)$, this range is narrow on relative scale, and thus the cancellation is unlikely (see Fig. 3 below). As a result, large precautionary savings most likely render the interest rate both low and volatile.

Furthermore, interest rates are potentially volatile regardless of the direction of savings cyclicality. When $Q(w, t)<\frac{2 \mu^{w}}{\left(\sigma^{w}\right)^{2}}$, the volatility of intertemporal consumption smoothing dominates the precautionary savings term. Given a positive shock to endowment, the aggregate risk aversion decreases and the elasticity of intertemporal substitution increases; agents tend to defer more consumption to later time and the interest rate drops. In other words, the equilibrium interest rate is countercyclical in this case. Conversely, when $Q(w, t)>\frac{2 \mu^{w}}{\left(\sigma^{w}\right)^{2}}+R(w, t)$, the volatility of precautionary savings dominates the consumption smoothing term. Given a positive shock to endowment, the precautionary savings term decreases and the interest rate surges. In other words,

\footnotetext{
${ }^{16}$ That is, the lower bound of interest rate volatility holds for most values of the savings motive cyclicality $Q$ as specified in this proposition.
} 
the interest rate is procyclical here 17 We can also draw parallel results from related literature. Kimball $(1992,1993)$ finds in a partial equilibrium model that sufficiently temperate (large positive $Q$ ) investors may invest most of their savings in safe assets. Our findings on the relation between temperance and interest rate volatility echo this link in general equilibrium settings.

Quantitatively, the lower bound of interest rate volatility is substantial when $Q$ is not in the vicinity of a knife-edge (critical) value of $\frac{2 \mu^{w}}{\left(\sigma^{w}\right)^{2}}$. For sufficiently large precautionary savings $P$ 25 (to render a low interest rate), when $Q$ is slightly off from the above critical value, the lower bound is several times larger than the observed interest rate volatility of $2 \%$ (Table 1 )

$$
\frac{\left|Q(w, t)-\frac{2 \mu^{w}}{\left(\sigma^{w}\right)^{2}}\right|}{\frac{2 \mu^{w}}{\left(\sigma^{w}\right)^{2}}}>0.1 \longrightarrow\left|\sigma_{\Gamma}^{r}\right|>0.1 R(w, t) \frac{2\left(\mu^{w}\right)^{2}}{\sigma^{w}}>6 \%,
$$

where the last numerical value is based on a conservative Hansen-Jagannathan bound (27). Fig. 3 illustrates this bound in a setting with two heterogeneous CRRA agents. The figure plots the volatility of interest rate (upper panel) vis-a-vis the cyclicality of precautionary savings motives as characterized by temperance $Q\left(p^{A}\right)$ (lower panel). The choice of risk aversion parameters $\left\{\gamma^{A}, \gamma^{B}\right\}$ are dictated by the low empirical interest rate and Hansen-Jagannathan bound (25), (27). As stated by proposition 5, we clearly see that interest rate volatility is small only when temperance $Q$ assumes values in the immediate vicinity of the critical value $Q^{*}=\frac{2 \mu^{w}}{\left(\sigma^{w}\right)^{2}}\left(\right.$ or $\left.p^{A} \approx 0.35\right)$. When $Q$ is slightly off this value (by a few percentage points), the interest rate is hugely volatile 18

Proposition 5 underlines the rich and complex equilibrium dynamics of the heterogeneous economy. It shows, for e.g., that a standard cure addressing, say, the level of the rfr may adversely increase its volatility. All that said, though large precautionary savings motive has been found very useful in addressing the equity premium and interest rate level in literatures, it is likely to bring about an unrealistically volatile rfr in the heterogeneous-agent economies (with additive utilities). The incompatibility of these canonical exchange economies and the observed equity premium is well known 19 Our contribution here is to offer a new analytical perspective on this incompatibil-

\footnotetext{
${ }^{17}$ Detailed portfolio choice solutions for multiple-agent economies with general additive utilities, as considered in proposition (5), are beyond the scope of this paper. Their closed-form expressions are not known and may not exist.

${ }^{18}$ Note that $\left.Q\left(p^{A}\right)=Q^{*}=\frac{2 \mu^{w}}{\left(\sigma^{w}\right)^{2}}\right)$ in another region in the vicinity of $p^{A}=1$, where interest rate is both low and smooth. But in this region the less risk averse agent $A$ dominates the economy, hence Hansen-Jagannathan bound is strongly violated, and stock market is incorrectly priced by the model.

${ }^{19}$ New elements in preferences such as habit formation (Campbell and Cochrane (1999)), catching-up-with-theJoneses (Chan and Kogan (2002)), or recursive utility together with growth rate long-run predictability (Bansal and
} 

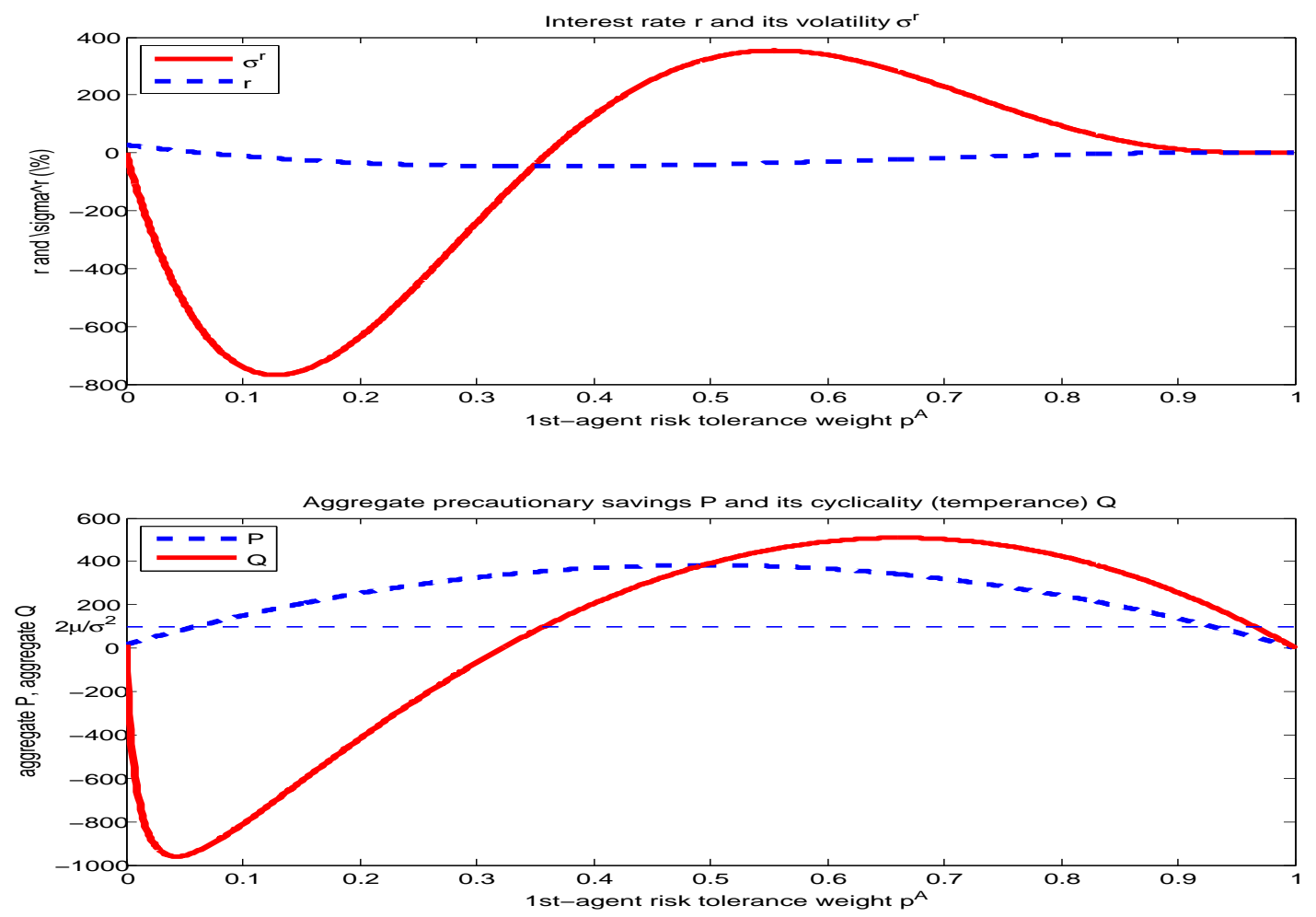

Figure 3: Two-CRRA-agent endowment economy: $\gamma^{A}=0.01, \gamma^{B}=15, \mu^{w}=2 \%, \sigma^{w}=2 \%$. The upper panel plots the interest rate $r\left(p^{A}\right)$ and interest rate volatility $\sigma^{r}\left(p^{A}\right)$ in $\%$, the lower panel plots the market-revealed (aggregate) precautionary savings motive (prudence) $P\left(p^{A}\right)$ (eq. 18) and savings cyclicality (temperance) $Q\left(p^{A}\right)$ (eq. $(22)$ ), and $Q\left(p^{A}\right)-P\left(p^{A}\right)-1$. These are ploted against agent $A^{\prime}$ 's risk tolerance weight $p^{A}=\frac{T^{A}}{T^{A}+T^{B}}=\frac{c^{A} \gamma^{B}}{c^{A} \gamma^{B}+c^{B} \gamma^{A}}$.

ity, within the standard setting of time separable preferences.

We next consider adding heterogeneity in time preferences to see whether that can ease the puzzles. The contribution of time preference heterogeneity can be computed either directly, as to be performed in this section, or indirectly by first homogenizing this heterogeneity, as explained in section 6. The component $\sigma_{\Delta}^{r}$ of rfr volatility (28) arises from an interesting interaction between

Yaron (2004)) have been invoked to tackle these asset price puzzles. In a new hybrid approach, Lettau and Wachter (2009) enlarge the state variable space to include exogenous short rate process while maintaining the equilibrium-based relation between the market price of risk and the fundamental dividend process. 
heterogeneities in risk aversion and discount factors

$$
\sigma_{\Delta}^{r} \equiv w \sigma^{w} \delta_{w}(w, t)=\sigma^{w} R(w, t) \operatorname{Cov}_{\left\{p^{i}\right\}}\left(T_{c}^{i}, \delta^{i}\right)
$$

where the last equality is an application of proposition 2 , also derived in appendix A (eq. (64)). The covariance structure is rich because both the risk tolerance measure $\left\{p^{i}\right\}$ and marginal risk tolerance $T_{c}^{i}$ are dynamic. In a CRRA economy, the latter is the inverse of the risk aversion coefficient. In that setting, the sign of $\sigma_{\Delta}^{r}$ depends on the relative orderings (comonotone or anti-comonotone) between risk aversions $\left\{\gamma^{i}\right\}$ and discount factors $\left\{\delta^{i}\right\}$. Under a positive supply shock $d w>0$, a procyclical discount factor $\delta_{w}>0$ increases the time value of consumption, thus encourages consumption and discourages savings. It thereby leads to a surge in the $\mathrm{rfr} r$. Hence, a procyclical discount factor contributes to procyclicality in interest rates and vice versa. The heterogeneity in time preferences can have either positive or negative effect on rfr volatility, and therefore can help temper the extreme nature of the latter's bound.

Indeed, combining (28), (32) and (34) yields more comprehensive bounds on rfr volatility

$$
\begin{array}{ll}
\sigma^{r}(w, t)>\sigma^{w} R(w, t)\left(\mu^{w} Q(w, t)-\frac{2\left(\mu^{w}\right)^{2}}{\left(\sigma^{w}\right)^{2}}+\operatorname{Cov}_{\left\{p^{i}\right\}}\left(T_{c}^{i}, \delta^{i}\right)\right) & \text { if } \quad Q(w, t)>\frac{2 \mu^{w}}{\left(\sigma^{w}\right)^{2}}+R(w, t), \\
\sigma^{r}(w, t)<\sigma^{w} R(w, t)\left(\mu^{w} Q(w, t)-\frac{2\left(\mu^{w}\right)^{2}}{\left(\sigma^{w}\right)^{2}}+\operatorname{Cov}_{\left\{p^{i}\right\}}\left(T_{c}^{i}, \delta^{i}\right)\right) & \text { if } \quad Q(w, t)<\frac{2 \mu^{w}}{\left(\sigma^{w}\right)^{2}} .
\end{array}
$$

Specifically, for countercyclical precautionary savings motive $Q(w, t)<\frac{2 \mu^{w}}{\left(\sigma^{w}\right)^{2}}$, a time preference ordering such that $\operatorname{Cov}_{\left\{p^{i}\right\}}\left(T_{c}^{i}, \delta^{i}\right)>0$ helps loosen the bound on the volatility of the interest rate ${ }^{20}$ Similar condition holds for the other case where $Q(w, t)>\frac{2 \mu^{w}}{\left(\sigma^{w}\right)^{2}}+R(w, t)$. Despite being a function of consumption allocations $\left\{c^{i}\right\}$, the covariance term is intimately associated with the discount rate heterogeneity structure, and can be formulated largely independent of the temperance term in $35{ }^{21}$ This makes heterogeneity in time preference a venue to mitigate the interest rate volatility in the consumption-based pricing models. In an attractive alternative approach, Garleanu and Panageas (2010) show that the combined features of overlapping generations and heterogeneous preferences are able to sustain the long-term survival of groups with different risk aversions, while

\footnotetext{
${ }^{20}$ In CRRA settings, $T_{c}^{i}=1 / \gamma^{i}$, so $\operatorname{Cov}_{\left\{p^{i}\right\}}\left(T_{c}^{i}, \delta^{i}\right)=\operatorname{Cov}_{\left\{p^{i}\right\}}\left(\frac{1}{\gamma^{i}}, \delta^{i}\right)>0$. This means that small $\gamma^{i}$ are most likely associated with large $\delta^{i}$ and vice versa (anti-comonotone). These are configurations wherein no agent dominates other in long run (see section 6).

${ }^{21}$ In CRRA settings, the covariance term is always negative if RRAs and discount factors are co-monotone $\left(\gamma^{i}>\right.$ $\left.\gamma^{j} \leftrightarrow \delta^{i}>\delta^{j}\right)$, and positive if anti co-monotone $\left(\gamma^{i}>\gamma^{j} \leftrightarrow \delta^{j}>\delta^{i}\right)$, independent of consumption dynamics.
} 
generating stable risk-free rate.

\section{$5 \quad$ Equity return volatility}

How do heterogeneities in risk and time preferences affect the volatility of return on stock? The answer is considerably more involved than that for the interest rate because the stock price $S$ is a contingent claim on the entire series of future dividend streams. To pursue this question, we employ the convenient tool of Malliavin calculus, following closely the approach presented in Detemple et. al. (2003) and Bhamra and Uppal (2009). We assume that there are just two classes, $A$ and $B$, of CRRA agents, thus simplifying the exposition while retaining heterogeneity. In such economies, there is a single state variable, which can be chosen as agent $A$ 's risk tolerance weight $p^{A}=\frac{T^{A}}{T}$. Detailed derivations can be found in the appendix B.

In risk-neutral measure $\mathcal{Q}$, all payoffs are discounted at the risk-free rate $r$. The stock price then is

$$
S(w, t)=e^{\int_{0}^{t} r(u) d u} E_{t}^{\mathcal{Q}}\left[\int_{t}^{T} e^{-\int_{0}^{u} r(\tau) d \tau} w(u) d u\right]
$$

In our Markovian (GBM) setting, the stock price $S(w, t)$ is a function of current endowment $w$, and thus stock return volatility $\sigma^{s}$ can be defined from the associated diffusion process (i.e., gain process)

$$
\frac{d S(w, t)+d w}{S(w, t)}=\mu^{s}(w, t) d t+\sigma^{s}(w, t) d Z(t)
$$

A standard application of Malliavin calculus confirms these relations

$$
\sigma^{s}(w, t)=\sigma^{w}-\frac{B(w, t)}{S(w, t)} E_{t}^{\mathcal{Q}}\left[\int_{t}^{T} d u \frac{w(u)}{B(w, u)} \int_{t}^{u} d \tau \mathcal{D}_{t}\left(\sigma^{w} \eta(w, \tau)+r(w, \tau)\right)\right]
$$

where $B(w, t)=\exp \left(\int_{0}^{t} r(w, u) d u\right)$ is the numeraire associated with the money market account, and $\mathcal{D}_{t}$ denotes the Malliavin derivative at time t. This representation of stock return volatility is very intuitive, as it reflects fluctuations both in the fundamental dividend and the discounting process. In the deterministic discounting scheme $(r, \eta$ are constant), fluctuation in the stock return results entirely and without distortion ${ }^{22}$ from stochastic movement in the dividend process $\sigma^{s}=\sigma^{w}$. However, in the current general equilibrium settings, both the interest rate and the market

\footnotetext{
${ }^{22}$ Note that the volatility $\sigma^{w}$ of GBM endowment is kept constant by construction.
} 
price of risk are endogenous and stochastic. They then also contribute to the excess volatility $\sigma^{s}(w, t)-\sigma^{w}$ (terms $\sigma^{w} \mathcal{D}_{t} \eta$ and $\left.\mathcal{D}_{t} r\right)$ in $(38)$ ) of the stock return via the discounting mechanism. Because the Malliavin derivative of a process $X$ is proportional to its volatility $\sigma^{X}: \mathcal{D}_{t} X \sim \sigma^{X}$ (see (80)), we arrive at a simple sufficient condition for stock return excess volatility to be positive, $\sigma^{s}(w, t)-\sigma^{w}>0$, in the current two-CRRA-agent economy

$$
\frac{\partial}{\partial p^{A}(\tau)}\left[r\left(p^{A}, \tau\right)+\sigma^{w} \eta\left(p^{A}, \tau\right)\right]<0
$$

Empirically, the return excess volatility in stock market is pointed out first by Shiller (1981). Here the above condition allows us to rigorously validate intuitive arguments from the consumption CAPM literature attempting to address this anomaly. In particular, either a countercyclical Sharpe ratio or a countercyclical rfr acts to boost the stock return volatility. We now discuss these two components in more detail.

All else equal, when the interest rate $r$ is countercyclical, $r$ and hence the discount rate decrease with the output. Similarly, when the Sharpe ratio $\eta$ is countercyclical, the risk premium, and again the discount rate, also tend to move in opposite direction with the supply. Given a positive shock to the endowment, the contingent claim (stock) price plausibly increases. However, under either countercyclical $r$ or $\eta$, the stock price would increase more than proportionally with the endowment because the discount rate tends to drop in both cases as mentioned above. The opposite holds when the endowment shock is negative. This is why either a countercyclical Sharpe ratio $\sigma^{\eta}<0$ or countercyclical interest rate $\left(\sigma^{r}<0\right)$ would contribute directly to positive stock return excess volatility $\sigma^{s}(w, t)-\sigma^{w}$, as expressed by each component of (39). The countercyclicality is a feature present in many models in the equity premium literature, and is pivotal to producing empirical patterns of predictability in stock returns. Campbell and Cochrane (1999) enlist habit formation to generate a Sharpe ratio that is high when aggregate consumption is low and vice versa. Chan and Kogan (2002) construct a heterogeneous-agent economy with a catching-up-withthe-Joneses feature in preferences, which renders risk premia countercyclical to endowment shocks. Quantitatively, a standard Ito manipulation on $\eta$ (24) yields the following Sharpe ratio volatily (with the convention: $\left.d \eta(w, t)=\mu^{\eta}(w, t) d t+\sigma^{\eta}(w, t) d Z(t)\right)$

$$
\sigma^{\eta}(w, t)=w\left(\sigma^{w}\right)^{2} R_{w}(w, t)
$$


It follows that the condition $\sigma^{\eta}(w, t)<0$ is achieved, as one would expect, when market-revealed risk aversion is decreasing with respect to aggregate consumption, $R_{w}(w, t)<0$. This is behaviorally quite reasonable as we would expect agents to be bolder in accommodating risks when they are richer. As viewed intuitively and generically as a direct implication of the risk sharing mechanism (proposition 2), a negative $R_{w}$ originates from the dynamics of the risk tolerance measure, which favors less risk averse agents after a positive shock to the endowment, and vice versa. It thus arises very naturally in the setting with heterogeneous CRRA agents (see (12) and also Wang (1996)). In a more general setting (beyond the CRRA framework), this countercyclicality is easily observed under the premise of large precautionary saving (25). Indeed, we can use (17) to rewrite $\sigma^{\eta}(w, t)$ in terms of the aggregate characteristics $R(w, t), P(w, t)$

$$
\sigma^{\eta}(w, t)=\left(\sigma^{w}\right)^{2} R(w, t)[1+R(w, t)-P(w, t)]
$$

Unless $R$ assumes unreasonably large values, $R>P>\frac{2 \mu^{w}}{\left(\sigma^{w}\right)^{2}} \sim 100$, the condition on large savings 25. needed for a low interest rate readily assures a countercyclical Sharpe ratio. Alternatively, proposition 6 below provides an agent-based sufficient condition for the countercyclicality beyond CRRA framework.

Proposition 6 When all agents' risk aversions and precautionary savings motives satisfy the relation $P^{i}\left(c^{i}, t\right) \geq 1+R^{i}\left(c^{i}, t\right)$ on the equilibrium consumption path $\left\{c^{i}\right\}_{i}$, the counterpart relation must hold at the aggregate level: $P(w, t) \geq 1+R(w, t)$.

Intuitively, given a certain degree of uniformity among the heterogeneous agents, this proposition asserts that the individual preference properties, that are central to determining the price volatilities, are preserved under dynamic aggregation. In other words, when all agents possess a large precautionary savings motive, so does the economy as a whole. Proposition 6 confirms and states this intuition as a rigorous sufficient condition. Whereas the risk aversion aggregation is linear (proposition 1), the aggregation on precautionary savings is highly nonlinear. This contrast makes these results far from obvious. It is also interesting to note that, $R_{w}(t, w)=\frac{R(t, w)}{w}(1+R(t, w)-P(t, w))$ as in (17), proposition 6 simply states that market-revealed risk aversion is decreasing in consumption if that property holds for each individual agent. A known special result of this proposition is obtained when all individual utilities belong to the CRRA class, whence both $R^{i}=\gamma^{i}, P^{i}=\gamma^{i}+1$ 
are constant and satisfy the hypothesis of proposition 6. Then

$$
P(w, t)=R(w, t)+1-\operatorname{Cov}_{\left\{p^{i}\right\}}\left(\gamma^{i}, \frac{1}{\gamma^{i}}\right)>R(w, t)+1
$$

Proposition 6, however, holds more generally for any additive expected utilities.

Back to the condition (39); combining its two terms yields a more complete insight into the relation between stock price movement and the economy's behavior toward risks. We rewrite this sufficient condition for positive stock return excess volatility in term of aggregate quantities $R, P$, $Q$

$$
\left(\sigma^{w}\right)^{2}\left[\frac{\mu^{w}}{\left(\sigma^{w}\right)^{2}}(1+R(w, t)-P(w, t))+\frac{P(w, t)(Q(w, t)-R(w, t)-4)}{2}\right]<-\operatorname{Cov}_{\left\{p^{i}\right\}}\left(\delta^{i}, \frac{1}{\gamma^{i}}\right) .
$$

A few important observations should be made. First, each of risk aversion, precautionary savings and temperance affects stock return volatilities. Intuitively, this is because all three influence savings and portfolio choices. The mechanism at work is as follows. All else being equal, small $Q$ enforces the above sufficient condition, and therefore boosts the excess volatility of the return on stocks. We recall from $(20)$ that temperance $Q$ is crucially related to $P_{w}$, namely small enough $Q$ is associated with procyclical $P$. A positive supply shock will increase precautionary savings (as $P_{w}>0$ ), leading to a decrease in both the interest and discount rates (see (24)). Thus the stock price increases more than proportionally compared to the endowment, which implies excess volatility in the stock return. (See also Shiller (1981) for a behavioral explanation of this phenomenon.)

Second, the relative orderings between agents' risk aversions and subjective discount factors also influence return volatility, via the term $\operatorname{Cov}_{\left\{p^{i}\right\}}\left(\delta^{i}, \frac{1}{\gamma^{i}}\right)$. That is because these orderings determine the dynamics of risk sharing, consumption partition and risk tolerance measure in the economy. These in turn are compounded in the asset price movements due to changes in endowment. We will return to these heterogeneity effects in the next section.

Finally, it is noted that while risk aversion and the precautionary savings motive have enjoyed substantial credence as shapers of asset price patterns in consumption-based pricing models, the cyclical properties of precautionary savings (or equivalently, temperance) are not well studied. Our investigation makes explicit the important link between these cyclical properties and asset (bond and stock) return volatilities. One reason why this very intuitive link has been quite implicit in 
the literature lies with the heterogeneity structure of the model itself. For a close illustration, we consider the setting of Bhamra and Uppal (2009). They obtain the first sufficient condition for positive stock return excess volatility that involves solely precautionary savings ${ }^{23}$ How can we reconcile this result with our condition (42)? The answer is as follows. In the two-CRRA-agent economy, as seen earlier, there is only a single state variable. This can be chosen without loss of generality as the first agent's risk tolerance measure $p^{A}=\frac{T^{A}}{T}$. Each and every aggregate quantity $R, P$ and $Q$ then is a simple function of $p^{A}$, and thus they pairwise bear a one-to-one relation. ${ }^{24}$ The derivation of Bhamra and Uppal's sufficient condition exploits these simple relations, and in doing so inadvertently obscures the role of temperance $Q(w, t) 25$ In fact, by virtue of $(24)$, the derivative of $\operatorname{rfr} \frac{d r}{d p^{A}}$ contains the term $\frac{d P}{d p^{A}}=\frac{P_{w}}{p_{w}^{A}}$, which is obviously related to the cyclicality $P_{w}$ of precautionary savings. This example and $\sqrt{42}$ together indicate that in more general multipleagent settings $R$ and $P$ are important, but far from sufficient statistics to determine stock return volatilities.

It is reassuring that all the above observations and intuitions concerning the cyclicality of precautionary savings, or equivalently temperance, also underlie the parallel results on interest rate volatility, reported in proposition 5 .

\section{Heterogeneities and homogenization of beliefs}

The heterogeneous-agent economies we have explored so far address heterogeneities in risk aversion and time preferences. As we have seen, these differences can foster rich and resilient exchanges leading to the equilibrium when agents assume off-setting characteristics in their preferences. While a higher degree of patience (smaller $\delta^{i}$ ) favors deferring consumptions, a larger elasticity of intertemporal substitution $\psi^{i}$ (equivalently lower risk aversion $\gamma^{i}=\frac{1}{\psi^{i}}$ in the additive utility framework) produces the same effect. Another practical and important factor in which agents differ is in their subjective beliefs about economic fundamentals. Such beliefs directly affect agents' intertemporal

\footnotetext{
${ }^{23}$ Bhamra and Uppal (2009) investigates an exchange economy with two agents who differ only in risk aversion. Their proposition 2 presents a sufficient condition for positive stock return excess volatility; $P<1+\frac{\mu^{w}}{\left(\sigma^{w}\right)^{2}}$. This is a stronger version of $(42)$, when 42 is adapted to the setting of homogeneous time preferences.

${ }^{24}$ In two-CRRA-agent economy, we have $P(w, t)=R(w, t)\left(1+\frac{\gamma^{A}+\gamma^{B}-R(w, t)}{\gamma^{A} \gamma^{B}}\right)$.

${ }^{25}$ Since $P=\left(p^{A} \gamma^{A}+p^{B} \gamma^{B}\right)\left(1+\frac{p^{A}}{\gamma^{A}}+\frac{p^{B}}{\gamma^{B}}\right)$, we have $\frac{d P}{d p^{A}}=\left(\gamma^{A}-\gamma^{B}\right)\left(\frac{P}{R}-\frac{R}{\gamma^{A} \gamma^{B}}\right)$ (this relation is needed in the derivation of key condition $\left(39\right.$, see $(83)$ ). Thus $\frac{d P}{d p^{A}}$, and for that matter, sufficient condition 39 appear unrelated to temperance $Q$, while they actually are.
} 
decisions and thus asset prices. In this section we will show that, as far as consumption and risk sharing are concerned, an economy whose agents differ in all time preferences, risk aversions and beliefs may be transformed isomorphically into a far simpler one with heterogeneity only in risk aversion. The required transformation offers new quantitative perspectives on the above-mentioned tradeoff between different dimensions of heterogeneity. The analysis also relates neatly to the survival of market participants (a.k.a market selection) in the long run.

\subsection{Heterogeneity in time preferences, risk aversions and beliefs}

We consider the canonical case, widely studied in literature, of a two-CRRA-agent economy with GBM endowments. The next section addresses the setting with multiple agents. In addition to heterogeneities in discount factors and risk aversion, agents $A, B$ also differ in their beliefs about the growth rates $\mu^{A}, \mu^{B}$ of the endowment process $w(t)(1)$. The realizations of $w(t)$ are correctly observed by all parties

$$
\mu^{w, A} d t+\sigma^{w} d Z^{A}(t)=\frac{d w(t)}{w(t)}=\mu^{w, B} d t+\sigma^{w} d Z^{B}(t)
$$

where $Z^{A}(t), Z^{B}(t)$ are standard Brownian motions under each agent's subjective information set (i.e., belief). We assume agents act on their own persistent beliefs ${ }^{26}$ A comparison with (1) yields

$$
\begin{array}{ll}
d Z^{A}(t)=d Z(t)+\theta^{A} d t ; & \theta^{A}=\frac{\mu^{w}-\mu^{w, A}}{\sigma^{w}} \\
d Z^{B}(t)=d Z(t)+\theta^{B} d t ; & \theta^{B}=\frac{\mu^{w}-\mu^{w, B}}{\sigma^{w}}
\end{array}
$$

Coefficient $\theta^{i}$ in essence characterizes the deviation of agent $i$ 's beliefs on the endowment growth rate $\mu^{w, i}$ from the its true value $\mu^{w}$. When $\theta^{i}<0$, agent $i$ is optimistic (with respect to the objective growth rate $\mu^{w}$ ) and vice versa. Also, two agents assign different but equivalent probability measures and distributions to the future uncertain endowment process. Since agents are still allowed to trade in the riskless bond and a contingent claim on the aggregate endowment (stock), the market is complete and the equivalent-agent optimization problem can be constructed to explicitly account

\footnotetext{
${ }^{26}$ That is, agents do not draw inferences from the willingness to trade by others. Later, we will extend our framework to accommodate time-varying beliefs, which in turn may arise from learning or other ad-hoc belief adjustment mechanism.
} 
for different beliefs

$$
\begin{aligned}
& \max _{\left\{c^{A}(t), c^{B}(t)\right\}} \frac{1}{\lambda^{A}} E_{0}^{(A)}\left[\int_{0} e^{-\delta^{A} t} u^{A}\left(c^{A}\right) d t\right]+\frac{1}{\lambda^{B}} E_{0}^{(B)}\left[\int_{0} e^{-\delta^{B} t} u^{B}\left(c^{B}\right) d t\right] \\
& \text { s.t. } c^{A}(t)+c^{B}(t)=w(t) \quad \forall t .
\end{aligned}
$$

Here $u^{i}=\frac{\left(c^{i}\right)^{1-\gamma^{i}}}{1-\gamma^{i}}$, and $E_{t}^{(i)}[\ldots]$ denotes the time- $t$ conditional expectation under agent $i$ 's belief. There exists a standard approach (see e.g., Detemple and Murthy (1994) and Basak (2005)) to convert the above optimization problem to one under the physical measure

$$
\begin{aligned}
& \max _{\left\{c^{A}(t), c^{B}(t)\right\}} E_{0}\left[\frac{1}{\lambda^{A}} \int_{0} \xi^{A}(t) e^{-\delta^{A} t} u^{A}\left(c^{A}\right) d t+\frac{1}{\lambda^{B}} \int_{0} \xi^{B}(t) \theta(t) e^{-\delta^{B} t} u^{B}\left(c^{B}\right) d t\right] \\
& \text { s.t. } c^{A}(t)+c^{B}(t)=w(t) \quad \forall t .
\end{aligned}
$$

The above operation involves a change of measure, from subjective $\mathbb{P}^{i}$ to physical $\mathbb{P}$, using the Radon-Nikodym derivative $\xi^{i}(t)$

$$
\xi^{i}(t)=\frac{d \mathbb{P}^{i}}{d \mathbb{P}}=\exp \left(-\frac{1}{2}\left(\theta^{i}\right)^{2} t-\theta^{i} Z(t)\right) \quad i \in\{A, B\}
$$

where $\theta^{i}$ is given in 43 . The dynamics of this heterogeneous-agent economy is captured by the FOC and the market clearing equation

$$
\left\{\begin{array}{c}
\frac{1}{\lambda^{A}} e^{-\delta^{A} t} \xi^{A}(t)\left(c^{A}(t)\right)^{-\gamma^{A}}=\frac{1}{\lambda^{B}} e^{-\delta^{B} t} \xi^{B}(t)\left(c^{B}(t)\right)^{-\gamma^{B}} \\
c^{A}(t)+c^{B}(t)=w(t)
\end{array}\right.
$$

Here we clearly see that all three dimensions of heterogeneity - risk aversion, time preference and belief - play roles in shaping the equilibrium. To simplify the analysis, it would be desirable to reduce this economy to one where only risk aversion experiences heterogeneous. Remarkably, that is possible. Consider the following simple multiplicative transformation (which is derived in the proof of proposition 7 , see appendix CP

$$
\left\{\begin{array}{l}
c^{A}(t) \rightarrow \hat{c}^{A}(t) \equiv \Upsilon(Z(t), t) c^{A}(t) \\
c^{B}(t) \rightarrow \hat{c}^{B}(t) \equiv \Upsilon(Z(t), t) c^{B}(t) \\
w(t) \rightarrow \hat{w}(t) \equiv \Upsilon(Z(t), t) w(t)
\end{array}\right.
$$


where

$$
\begin{gathered}
\Upsilon(Z(t), t)=\exp \left(\beta^{\gamma, \delta} t\right) \exp \left(\beta^{\gamma, \theta} Z(t)\right) \\
\beta^{\gamma, \theta} \equiv \frac{\theta^{A}-\theta^{B}}{\gamma^{A}-\gamma^{B}} ; \quad \beta^{\gamma, \delta} \equiv \frac{\delta^{A}+\frac{\left(\theta^{A}\right)^{2}}{2}-\delta^{B}-\frac{\left(\theta^{B}\right)^{2}}{2}}{\gamma^{A}-\gamma^{B}} \equiv \frac{\delta_{\text {eff }}^{A}-\delta_{\text {eff }}^{B}}{\gamma^{A}-\gamma^{B}} .
\end{gathered}
$$

The coefficients $\delta_{\text {eff }}^{A} \equiv \delta^{A}+\frac{\left(\theta^{A}\right)^{2}}{2}, \delta_{\text {eff }}^{B} \equiv \delta^{B}+\frac{\left(\theta^{B}\right)^{2}}{2}$ are the effective discount rates of agent $A$ and $B$ respectively, with their subjective beliefs being incorporated. The coefficients $\beta^{\gamma, \theta}$ and $\beta^{\gamma, \delta}$ quantify respectively differences in beliefs and in time preferences, normalized with respect to the difference in risk aversions. These coefficients will have a neat interpretation as slopes of a linear projection in characteristics space $(\delta, \gamma, \theta)$ when we come to the full multiple-agent settings in the next section. Interestingly, we note that this transformation indeed considerably simplifies the full dynamics (46), which now become

$$
\left\{\begin{array}{c}
\frac{1}{\lambda^{A}}\left(\hat{c}^{A}(t)\right)^{-\gamma^{A}}=\frac{1}{\lambda^{B}}\left(\hat{c}^{B}(t)\right)^{-\gamma^{B}} \\
\hat{c}^{A}(t)+\hat{c}^{B}(t)=\hat{w}(t)
\end{array} .\right.
$$

Equation 49 represents the familiar dynamics of a two-CRRA-agent economy whose agents differ only in their risk aversions $\gamma^{A}, \gamma^{B}$, as studied in Benninga and Mayshar (2000), Dumas (1989) and Wang (1996). Effectively, we have been able to "rotate" the heterogeneities in subjective beliefs and discount factors away by changing the aggregate endowment $w(t)$ to $\Upsilon(Z(t), t) w(t)$. This in turn is equivalent to shifting the growth and volatility rates of the GBM endowment

$$
\begin{gathered}
\frac{d \hat{w}(t)}{\hat{w}(t)} \equiv \mu^{\hat{w}} d t+\sigma^{\hat{w}} d Z(t), \\
\sigma^{\hat{w}}=\sigma^{w}+\beta^{\gamma, \theta}, \\
\mu^{\hat{w}}=\mu^{w}+\beta^{\gamma, \delta}+\beta^{\gamma, \theta}\left(\sigma^{w}+\frac{\beta^{\gamma, \theta}}{2}\right) .
\end{gathered}
$$

Thus in the dynamics of consumption and risk sharing, the differences in time preferences and beliefs can be taken into account by modifying both the growth and volatility of the supply process. We will refer to $\left\{\gamma^{1}, \gamma^{2}, \delta^{1}, \delta^{2}, \theta^{1}, \theta^{2}, w(t)\right\}$ as the original economy, in which two CRRA agents differ in risk aversion, time preference and belief, as specified in 43 . Similarly, we denote $\left\{\gamma^{1}, \gamma^{2}, \hat{w}(t)\right\}$ as the reduced economy, whose agents differ only in risk aversion. The defining property of the transformation, that all agents' equilibrium consumptions stay the same up to a (stochastic) mul- 
tiplicative factor $\Upsilon(Z(t), t)$ in the two economies 47 , implies a profound relationship between the two respective consumption sharing dynamics. Not only are the consumption shares unchanged $\left(\frac{\hat{c}^{i}}{\hat{w}}=\frac{c^{i}}{w}\right.$ and $\left.\frac{\hat{c}^{i}}{\hat{c}^{j}}=\frac{c^{i}}{c^{j}}\right)$, but more importantly, the individual marginal propensities to consume out of the aggregate endowment (7), our key risk tolerance measure, remain identical in the two economies.

$$
\hat{c}_{\hat{w}}^{i}=\frac{\hat{T}^{i}\left(\hat{c}^{i}, t\right)}{\hat{T}(\hat{w}, t)}=\frac{\frac{\hat{c}^{i}}{\gamma^{i}}}{\sum_{i} \frac{\hat{c}^{i}}{\gamma^{i}}}=\frac{\frac{c^{i}}{\gamma^{i}}}{\sum_{i} \frac{c^{i}}{\gamma^{i}}}=\frac{T^{i}\left(c^{i}, t\right)}{T(w, t)}=c_{w}^{i} .
$$

And so do the aggregate characteristics built upon this measure in the two economies. The first is the (market-revealed) equivalent risk aversion (8)

$$
\hat{R}(\hat{w}, t)=\sum_{i} \frac{\hat{T}^{i}\left(\hat{c}^{i}, t\right)}{\hat{T}(\hat{w}, t)} \gamma^{i}=\sum_{i} \frac{T^{i}\left(c^{i}, t\right)}{T(w, t)} \gamma^{i}=R(w, t) .
$$

Market-revealed precautionary savings $P(w, t)$ and temperance $Q(w, t)$ are also identical in the two economies, which can be directly deduced from their expressions (71), (72) for CRRA utilities. Because of these relationships, we will refer to this key property generally as preserving consumption partition dynamics below. We summarize this precise correspondence in the following proposition.

Proposition 7 Suppose that the aggregate endowment follows a GBM process $w(t)$ (1), and that there are two classes of CRRA agents. In term of consumption partition dynamics at equilibrium, the two economies are isomorphic:

$$
\left\{\gamma^{1}, \gamma^{2}, \delta^{1}, \delta^{2}, \theta^{1}, \theta^{2}, w(t)\right\} \longleftrightarrow\left\{\gamma^{1}, \gamma^{2}, \hat{w}(t)\right\}
$$

where the isomorphic endowment $\hat{w}$ is also a GBM process defined in (50).

Though this result holds exactly under the specific premise of GBM endowment, it clearly shows the direction and possibility of an interesting and qualitative tradeoff between agent-based characteristics and aggregate supply statistics in more general cases. In this way, the findings in a reduced economy can be adapted to economies with additional dimensions of heterogeneity. Among others, the analytical results on the linkage between risk sharing and the size of endogenous credit markets obtained in Longstaff and Wang (2009) can be immediately generalized to allow agents to differ also in time preference. To fix the convention for the next discussion, we assume without loss of 
generality that $\gamma^{A}<\gamma^{B}$ throughout.

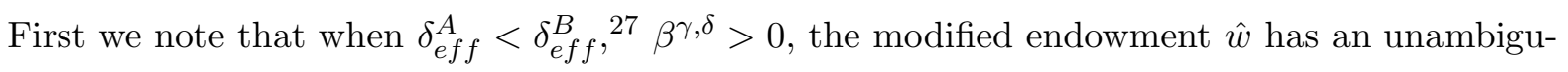
ously higher growth rate $(50)$. That is, as agent $A$ is both less risk averse and effectively more patient in the original economy, she would take more risk and be more willingly to defer consumption than would agent $B$. Then it is necessary to boost the isomorphic economy's endowment growth rate, in which agents are now equally patient ${ }^{28}$ to induce agent $A$ to undertake similar consumption sharing in equilibrium. The opposite holds when $\delta_{\text {eff }}^{A}>\delta_{\text {eff }}^{B}$. Second, when $\theta^{A}<\theta^{B}$, $\beta^{\gamma, \theta}>0$, the modified endowment $\hat{w}$ has both higher growth rate and volatility (50). That is, as agent $A$ is both less risk averse and more optimisti 29 in the original economy, she would bear risk more aggressively in this case too. Then to preserve equilibrium consumption partition dynamics, it is necessary to boost both the isomorphic economy's endowment growth rate and its volatility, given that agents now have identical beliefs. Finally, we also note that while time preference heterogeneity is reflected only in the isomorphic economy's endowment growth rate, belief heterogeneity influences both that growth rate and volatility. This is because a subjective belief relative to truth, as characterized by a Radon-Nikodym change of measure (45), is always stochastic, while a discount process $e^{-\delta^{i} t}$ is deterministic.

\section{Time-varying beliefs}

Interestingly, the above isomorphism also exists in the richer class where beliefs vary over time as agents observe the realizations of the endowment process. The analysis can address general forms of time variation of subjective beliefs, for which the perceived growth rates $\mu^{w, A}, \mu^{w, B}$ of endowment are bounded, adapted processes 30 Important special cases would be Bayesian updating and other ad-hoc learning mechanisms. In such settings, in place of (45), individual beliefs are characterized by the path-dependent Radon-Nikodym derivatives

$$
\xi^{i}(t)=\frac{d \mathbb{P}^{i}}{d \mathbb{P}}=\exp \left(-\frac{1}{2} \int^{t}\left(\theta^{i}(w, s)\right)^{2} d s-\int^{Z(t)} \theta^{i}(w, s) d Z(s)\right) \quad i \in\{A, B\} .
$$

\footnotetext{
${ }^{27}$ Since $\delta_{\text {eff }}^{A}=\delta^{A}+\frac{\left(\theta^{A}\right)^{2}}{2}, \delta_{\text {eff }}^{B}=\delta^{B}+\frac{\left(\theta^{B}\right)^{2}}{2}$, this inequality can be result of $\left\{\delta^{A}<\delta^{B} ; \theta^{A}=\theta^{B}\right\}$, or $\left\{\delta^{A}=\right.$ $\left.\delta^{B} ; \theta^{A}<\theta^{B}\right\}$, or some of their appropriate mixtures

${ }^{28}$ They are now heterogeneous only in risk aversions

${ }^{29} \theta^{A}<\theta^{B}$ and $\sqrt{43}$ imply that agent $A$ believes in a higher growth rate than agent $B: \mu^{w, A}>\mu^{w, B}$

${ }^{30}$ These are prerequisites for Girsanov's theorem on change of measure to work. See, e.g., section 3.5 in Karatzas and Shreve (1991).
} 
The coefficients $\theta^{A}, \theta^{B}$ now are bounded, adapted stochastic processes and describe possible evolution patterns of beliefs. To illustrate, let us briefly consider two examples. The first is the Bayesian updating case where agents' priors about the endowment's unobserved growth rate $\mu^{w}$ are normal distributions $N\left(m^{I}(t), v^{I}(t)\right), I \in\{A, B\}$. In this setting, Brennan (1998) obtains the following learning dynamic 31

$$
\left\{\begin{array}{l}
d m^{I}=\frac{v^{I}(0)}{v^{I}(0) t+\left(\sigma^{w}\right)^{2}}\left[\left(\mu^{w}-m^{I}\right) d t+\sigma^{w} d Z(t)\right], \quad I \in\{A, B\} . \\
v^{I}(t)=\frac{v^{I}(0)\left(\sigma^{w}\right)^{2}}{v^{I}(0) t+\left(\sigma^{w}\right)^{2}}
\end{array}\right.
$$

Evidently, as time lapses, both agents' beliefs converge to truth; $\lim _{t \rightarrow \infty} v^{I}(t) \rightarrow 0, \lim _{t \rightarrow \infty} m^{I}(t) \rightarrow$ $\mu^{w}, I \in\{A, B\}$. In the second example, even if agents eventually learn the truth, their beliefs may diverge incrementally following a negative shocks to the output when relation $\frac{\partial\left|\theta^{A}-\theta^{B}\right|}{\partial w}<0$ holds.

The current general belief heterogeneity can be rotated away by modifications in the growth and volatility of endowment process, similar to 47). The only difference with (48) is that now the transformation parameters $\beta^{\gamma, \theta}, \beta^{\gamma, \delta}$ are stochastic. Accordingly, in place of 50 , the endowment process of the isomorphic economy becomes

$$
\begin{gathered}
\frac{d \hat{w}(t)}{\hat{w}(t)} \equiv \mu^{\hat{w}} d t+\sigma^{\hat{w}} d Z(t), \\
\sigma^{\hat{w}}(w, t)=\sigma^{w}+\frac{\theta^{A}(w, t)-\theta^{B}(w, t)}{\gamma^{A}-\gamma^{B}}, \\
\mu^{\hat{w}}(w, t)=\mu^{w}+\frac{\delta^{A}-\delta^{B}}{\gamma^{A}-\gamma^{B}}+\frac{1}{2} \frac{\left[\theta^{A}(w, t)\right]^{2}-\left[\theta^{B}(w, t)\right]^{2}}{\gamma^{A}-\gamma^{B}}+\frac{\theta^{A}(w, t)-\theta^{B}(w, t)}{\gamma^{A}-\gamma^{B}}\left(\sigma^{w}+\frac{1}{2} \frac{\theta^{A}(w, t)-\theta^{B}(w, t)}{\gamma^{A}-\gamma^{B}}\right) .
\end{gathered}
$$

While the original output $w(t)$ is a pure geometric brownian process, its isomorphic counterpart $\hat{w}(t)$ incorporating the time variance in belief dynamics, generally belongs to richer classes. In particular, when beliefs diverges in bad time $(d w<0)$, the volatility of the isomorphic economy's endowment $\sigma^{\hat{w}}$ gets further away from that of the original economy $\sigma^{w}$, though the former economy does not necessarily become more volatile (i.e., $\sigma^{\hat{w}}$ can either increase or decrease with $w$ ). Furthermore, certain time-varying patterns of beliefs in the original economy may transform into a degree of mean reversion in the output of the isomorphic economy so that the risk-sharing dynamic between agents is preserved despite beliefs being homogenized. The mean reversion in the output's growth benefits alternatively one or the other agent when the trend turns ${ }^{32}$ This implies that the original

\footnotetext{
${ }^{31}$ We assume that agents agree to disagree, and learn only from the observed realizations of endowment.

${ }^{32}$ We will analyze in section 6.3 how the output's growth rate affects agents' survival in the long run.
} 
belief heterogeneity acts to compensate agents' difference in risk aversions in a way that sustain their presence in equilibrium, despite market selection. Qualitatively, the isomorphic transformation allows us to see quickly how heterogeneities in beliefs and time preferences affect agent's risk-sharing behaviors in the original economy per se. The dynamic (51) of isomorphic economy's output then initiates a quantitative analysis of the risk sharing in the simplified setting of heterogeneity only in risk aversion.

So far our analysis has involved two-CRRA-agent economies, for which case the isomorphism exists. We turn next to the more general setting with multiple CRRA agents and relate it naturally to the important issue of long-run survival of these agents.

\subsection{Multi-agent setting}

We now generalize the findings of the previous section to the case of many CRRA agents, and relegate missing derivations to the appendix C. Quantitatively, the consumption dynamics isomorphism between the original (fully heterogeneous) and the reduced (agents heterogeneous only in risk aversions) economy $\left\{\left\{\gamma^{i}, \delta^{i}, \theta^{i}\right\}_{i}, w(t)\right\} \longleftrightarrow\left\{\left\{\gamma^{i}\right\}_{i}, \hat{w}(t)\right\}$ is concerned with both FOC and market clearing.

$$
\left\{\begin{array} { c } 
{ \frac { 1 } { \lambda ^ { i } } e ^ { - \delta ^ { i } t } \xi ^ { i } ( t ) ( c ^ { i } ( t ) ) ^ { - \gamma ^ { i } } = M ( w , t ) \quad \forall i } \\
{ \sum _ { i } c ^ { i } ( t ) = w ( t ) }
\end{array} \longleftrightarrow \left\{\begin{array}{c}
\frac{1}{\lambda^{i}}\left(\hat{c}^{i}(t)\right)^{-\gamma^{i}}=\hat{M}(\hat{w}, t) \quad \forall i \\
\sum_{i} \hat{c}^{i}(t)=\hat{w}(t)
\end{array}\right.\right.
$$

In the above expressions, $M(w, t)$ and $\hat{M}(\hat{w}, t)$ are unique state price densities in the respective economies. The key to this isomorphism is the existence of a common multiplicative factor $\left(\Upsilon(Z(t), t)=\frac{\hat{c}^{i}}{\hat{c}}=\frac{\hat{w}}{\hat{w}} \forall i\right)$ that is able to absorb and homogenize all agent-specific time preferences and beliefs

$$
[\Upsilon(Z(t), t)]^{\gamma^{i}} e^{-\delta^{i} t} \xi^{i}(t)=\frac{M(w, t)}{\hat{M}(\hat{w}, t)} \forall i
$$

Plugging in agent $i$ 's belief $\xi^{i} 45$ for the GBM endowment under current consideration, the above condition is satisfied when two linear (quadratic) relations hold in characteristics space $\left(\delta^{i}, \gamma^{i}, \theta^{i}\right.$ ) 
( $A, B, C, D$ are some constants, that are identical for all agents)

$$
\left\{\begin{array}{c}
\delta_{e f f}^{i} \equiv \delta^{i}+\frac{\left(\theta^{i}\right)^{2}}{2}=A+B \gamma^{i}, \quad \forall i \\
\theta^{i}=C+D \gamma^{i}
\end{array}\right.
$$

Under these premises, much more meaningful interpretations can be obtained for coefficients $A, B, C, D$. Namely, they are the slope and intercept coefficients of projections from time preferences $\left\{\delta^{i}\right\}$ and beliefs $\left\{\theta^{i}\right\}$ onto risk aversion $\left\{\gamma^{i}\right\}$ parameter spaces.

$$
\begin{aligned}
& B=\beta^{\gamma, \delta}=\frac{\operatorname{Cov}\left(\gamma^{i}, \delta_{\text {eff }}^{i}\right)}{\operatorname{Var}\left(\gamma^{i}\right)}=\frac{\frac{1}{N} \sum_{i}^{N}\left(\gamma^{i} \delta_{\text {eff }}^{i}\right)-\frac{1}{N^{2}} \sum_{i}^{N} \gamma^{i} \sum_{j}^{N} \delta_{e f f}^{j}}{\frac{1}{N} \sum_{i}^{N}\left(\gamma^{i}\right)^{2}-\frac{1}{N^{2}}\left(\sum_{i}^{N} \gamma^{i}\right)^{2}} \\
& D=\beta^{\gamma, \theta}=\frac{\operatorname{Cov}\left(\gamma^{i}, \theta^{i}\right)}{\operatorname{Var}\left(\gamma^{i}\right)}=\frac{\frac{1}{N} \sum_{i}^{N}\left(\gamma^{i} \theta^{i}\right)-\frac{1}{N^{2}} \sum_{i}^{N} \gamma^{i} \sum_{j}^{N} \theta^{j}}{\frac{1}{N} \sum_{i}^{N}\left(\gamma^{i}\right)^{2}-\frac{1}{N^{2}}\left(\sum_{i}^{N} \gamma^{i}\right)^{2}}
\end{aligned}
$$

where $N$ is the number of agents in the economy. In this result, heterogeneities in beliefs and time preferences are accounted for by a change in endowment, very much like the setting with two agents

$$
\begin{gathered}
\Upsilon(Z(t), t)=\exp \left(\beta^{\gamma, \delta} t\right) \exp \left(\beta^{\gamma, \theta} Z(t)\right) \\
w(t) \longrightarrow \hat{w}(t)=\Upsilon(Z(t), t) w(t) \equiv \exp \left[\left(\mu^{\hat{w}}-\frac{\left(\sigma^{\hat{w}}\right)^{2}}{2}\right) t+\sigma^{\hat{w}} Z(t)\right], \\
\sigma^{\hat{w}}=\sigma^{w}+\beta^{\gamma, \theta} \\
\mu^{\hat{w}}=\mu^{w}+\beta^{\gamma, \delta}+\beta^{\gamma, \theta}\left(\sigma^{w}+\frac{\beta^{\gamma, \theta}}{2}\right) .
\end{gathered}
$$

In particular, when either $\gamma^{i}$ and $\theta^{i}$ (or $\gamma^{i}$ and $\delta_{\text {eff }}^{i}$ ) are co-monotone, the slope coefficients $\beta^{\gamma, \theta}$ (or $\beta^{\gamma, \delta}$ ) are positive. Then the growth rate $\mu^{\hat{w}}$ and volatility $\sigma^{\hat{w}}$ of the isomorphic endowment $\hat{w}$ are unambiguously larger than their original counterparts $\mu^{w}, \sigma^{w}$. This is because the comonotonicity in $\gamma^{i}$ and $\theta^{i}$ means agents are highly polarized; less risk averse agents are also likely more optimistic ones and vice versa. To induce agents to preserve their consumption sharing dynamics, it is necessary to boost both the growth rate and volatility of the endowment in the reduced economy, in which agents by construction have homogeneous time preferences and beliefs (that is, they are less polarized). The same applies for co-monotonicity in $\gamma^{i}$ and $\delta_{\text {eff }}^{i}$. These general intuitions, when combined with the regression-based interpretation of the coefficients $B, D$ in (54), 55), point again to the interesting tradeoff between microscopic (agent-based) characteristics and 
macroscopic (aggregate) supply statistics in the multiple-agent economy. When the the linearities (53) in characteristics space $\left(\delta^{i}, \gamma^{i}, \theta^{i}\right)$ do not hold, no exact isomorphism can be found between

the original $\left\{\left\{\gamma^{i}, \delta^{i}, \theta^{i}\right\}_{i}, w(t)\right\}$ and the reduced $\left\{\left\{\gamma^{i}\right\}_{i}, \hat{w}(t)\right\}$ economies. Nevertheless, the latter can always be explicitly constructed about the linear projections (54), (55) from time preferences $\left\{\delta^{i}\right\}$ and beliefs $\left\{\theta^{i}\right\}$ onto risk aversion $\left\{\gamma^{i}\right\}$, as we see in (57). We reasonably expect that the consumption partition dynamics in the reduced economy, heterogeneous only in risk aversions, would most closely match that of the original economy, heterogeneous in all three dimensions of risk aversion, time preference and beliefs.

So far in this section, our strategy for analyzing heterogeneous-agent economies has been to deform the aggregate supply process to the point that it fully (or best) accounts and thus compensates for agents' heterogeneities in time preferences and beliefs. In certain aspects, this pairs well with a popular strategy in the literature to substitute different dimensions of heterogeneity, either at the individual agent or representative agent level. The latter strategy addresses whether the risk loving, patience and optimism of each agent or the whole economy (market-revealed agent) are equivalent and mutually substitutable given observed risk sharing and price dynamics. In the single-generation settings under current consideration, a specific but central question is on the domination and survival of some agents over the others in the long run. Working in the context of the market selection, we now formally relate these two strategies.

\subsection{Agent survival}

Following Sandroni (2000) and Yan (2008) we use original economy's FOC (52) to examine the scaled equilibrium consumption ratio of any two agents $i, j$

$$
\begin{gathered}
\frac{\left[\frac{c^{i}(w, t)}{w(t)}\right]^{\gamma^{i}}}{\left[\frac{c^{j}(w, t)}{w(t)}\right]^{\gamma^{j}}}=\frac{\frac{1}{\lambda^{i}} e^{-\delta^{i} t} \xi^{i}(t)[w(t)]^{-\gamma^{i}}}{\frac{1}{\lambda^{j}} e^{-\delta^{j} t} \xi^{j}(t)[w(t)]^{-\gamma^{j}}} \\
=\frac{\lambda^{j} w_{0}^{-\gamma^{i}}}{\lambda^{i} w_{0}^{-\gamma^{j}}} \exp \left[\left(I^{j}-I^{i}\right) t\right] \exp \left[\left(\theta^{j}+\gamma^{j} \sigma^{w}-\theta^{i}-\gamma^{i} \sigma^{w}\right) Z(t)\right],
\end{gathered}
$$


where $w_{0}$ is the initial value of endowment, and

$$
I^{i} \equiv \delta^{i}+\frac{\left(\theta^{i}\right)^{2}}{2}+\gamma^{i}\left(\mu^{w}-\frac{\left(\sigma^{w}\right)^{2}}{2}\right) \quad \forall i
$$

Consider the case $\mu^{w}>\frac{\left(\sigma^{w}\right)^{2}}{2}$ so that the economy is growing statistically. When $I^{i}<I^{j}$, Yan (2008) notes that the above scaled equilibrium consumption ratio (58) grows to infinity almost surely as $t \rightarrow \infty$. As the consumption ratio $\frac{c^{j}}{w} \in[0,1]$ is bounded, this necessarily implies that $\frac{c^{j}(w, t)}{w(t)} \rightarrow 0$ almost surely, or agent $j$ will fail to survive in the long run 33 For this reason, parameters $I^{i}$ are referred to as survival indices. By performing this pairwise comparative analysis for all agents in this growing economy, Yan (2008) obtains a necessary condition for long-run survival in this economy.

$$
\lim _{t \rightarrow \infty} \frac{c^{i}(w, t)}{w(t)} \neq 0 \Longrightarrow i \in \arg \min _{j}\left\{I^{j}\right\}
$$

Any agent $i$ who survives in the long run must have minimum survival index among all agents. Clearly, either high risk aversion (large $\gamma^{i}$ ), impatience (large $\delta^{i}$ ) or pessimism (large $\theta^{i}$ ) will contribute negatively to the market selection of an agent. On top of these, the economy's strong growth (large positive $\mu^{w}-\frac{\left(\sigma^{w}\right)^{2}}{2}$ ) also fastens the extinction process for those who are not fit to survive. This is because, the statistically growing economies do not reward these characteristics of "reservation" nature in the long run 34 We note that this condition however is not strictly sufficient for survival. Consider the case where there are several agents $i, j$ all having minimum index $I^{i}=I^{j}=I_{\text {min }}$. In the limit of $t \rightarrow \infty$, standard Brownian motion $Z(t) \rightarrow \pm \infty$ with equal probability (a well-known non-stationarity problem). (58) then implies additionally that only agents having extremum (minimum or maximum) value of $\theta^{j}+\gamma^{j} \sigma^{w}$ (among agents with minimum survival index) survive. This observation allows us to deduce a more elaborated set of necessary conditions, that also connect well with our analysis of the isomorphic economy. Namely, common to all agents $i$ who survive, there exist two constants $K, L$ such that

$$
\left\{\begin{array}{l}
\delta^{i}+\frac{\left(\theta^{i}\right)^{2}}{2}+\gamma^{i}\left(\mu^{w}-\frac{\left(\sigma^{w}\right)^{2}}{2}\right)=K, \quad \forall i . \\
\theta^{i}+\gamma^{i} \sigma^{w}=L,
\end{array} \quad \forall i\right.
$$

\footnotetext{
${ }^{33}$ Here any agent $i$ 's long-run survival definition is that his consumption ratio $\frac{c^{i}(w, t)}{w(t)}$ does not tend to zero in the limit of large $t$.

${ }^{34}$ For example, more risk-loving agents have lower EIS, defer more consumption and invest more in risky equity relatively. When economy grows steadfastly, the stock market pays off well, and these agents quickly dominate the economy. The rate of their ascent increases with the economy's growth rate.
} 
These necessary conditions are none other than the linearity sufficient conditions for the existence of the reduced economy. The immediate conclusion is that the set of survival agents implies the existence of the exact isomorphic economy. To put it in another way, ultimately all heterogeneousagent economies specified in this section can be exactly reduced to its simpler isomorphic version, when all agents differ only in their risk aversion 35 Furthermore, in this case the reduced economy's supply $\hat{w}$ turns out to be constant, which makes the analysis of co-surviving agents even simpler. In the not-so-long run, the isomorphism does not hold exactly because other agents (who ultimately perish) hang on. Nevertheless, in the current setting with additive utilities, Kogan et. al. (2009) show that these agents leave no lingering traces on price dynamics after their consumption shares become negligible. Then as discussed earlier, the linear projection construction (54), (55) will determine qualitatively the time preference and heterogeneous belief contributions, as well as significantly simplifying the analysis on consumption partition and perhaps the asset price dynamics of the original economy.

We thus show that agent survival implies the existence of an isomorphic economy. But is the converse true, i.e., does isomorphism also imply survival? We recall that isomorphism just requires that the original economy can be reduced to a simpler economy heterogeneous only in risk aversion. Obviously, the latter generally does not imply survival, because both (i) agents are still heterogeneous in risk aversion and (ii) its aggregate endowment $\hat{w}$ can be either growing or shrinking steadily. Thus survival is the stronger concept, and the existence of isomorphic economy does not imply the survival of different agents in general. Only in a special case where the transform $\Upsilon(Z(t), t)$ assumes some particular functional forms, does the isomorphism imply the survival of all agents.

\section{Conclusion}

Finance, and economics more generally, has made great progress utilizing the representative agent model. However, real world agents differ significantly in risk aversion, time preference and beliefs. Moreover, such differences strongly motivate the trades that are made on financial markets, and

\footnotetext{
${ }^{35}$ In this regard, the special case when only one agent survives is trivial, because he eventually consumes the whole aggregate endowment. For time separable utilities under consideration, the economy will converge to a single-agent economy in all aspects as shown by Kogan et. al. (2009).
} 
therefore the behaviors of asset prices.

We analyzed the savings and consumption choices for agents who differ in preferences and beliefs within an economy with a GBM endowment. These choices translate into aggregates, which in turn determine asset price behavior. The most significant results are two remarkable isomorphisms, which may greatly facilitate the study of economies composed of heterogeneous agents. First, when agents differ only in risk aversion, the economy behaves as if all agents were identical to a single market-equivalent agent with a derived level of risk aversion. Second, when agents differ in all of risk preferences, time preferences and beliefs about the future growth of the economy, the economy is equivalent to one where all agents differ merely in risk aversion. Combining these two results, despite three dimensions of heterogeneity, the economy operates as if it were homogeneous and composed only of the market-equivalent agent.

Surprisingly, the aggregates in the heterogeneous economy, such as the "observed" precautionary savings motive, can lie well outside the behaviors that would be observed were the economy composed of any possible one of its constituent types of agents. That is because the dynamic risk sharing and trading of assets among types as the economy incurs shocks are of a stochastic nature. Low real interest rates, equivalent to those observed in the real world, can be achieved with reasonable risk aversions for all individual agents, given that large aggregate precautionary savings motives are feasible in equilibrium. However, such large savings motives tend to imply large savings cyclicality, which in turn generates unrealistic levels of interest rate volatility. (We show that such volatility can be dampened by heterogeneity in time preference.) Savings cyclicality also influences stock prices and volatility, as is demonstrated.

To move from the heterogeneity in all of risk aversion, time preference and beliefs to those merely on risk aversion, that is to dramatically reduce the dimensions of the problem, requires merely modifying the mean and volatility of the endowment process. We expect this insight to make future investigations of heterogeneities much more tractable.

The risk tolerance measure proves to be an extraordinarily versatile tool quantifying how individuals share risk and how resulting aggregate behaviors response to growth shocks. The sensitivities to these shocks (i.e., derivatives) of risk tolerance reveal how agents are jostled in their weightings within the economy as uncertainties unfold. Conveniently, these derivatives prove to be simple functions of individuals' risk aversion, prudence and temperance. This property allows us 
to obtain interesting and analytical bounds on asset return volatilities.

The principal risk that we face in the modern economy, as we witnessed in recent years, is the movement of asset prices within the economy. This analysis traced how agents who differ on preferences and beliefs trade amongst themselves to simultaneously hedge against, capitalize on and generate such movements. Most important, it showed that those tracings prove tractable. 


\section{Appendix}

We recall that subscripts always denote partial derivatives; $f_{x} \equiv \frac{\partial f}{\partial x}$ throughout the paper.

\section{A Proofs concerning risk tolerance measure}

\section{A.1 Preliminary derivations}

Derivation of key eq. (7): Using FOC (5) we have

$$
v_{w w}=\frac{1}{\lambda^{i}} u_{c c}^{i} c_{w}^{i}
$$

Plugging FOC (5) and above eq. into the expression for market-revealed risk tolerance (6)

$$
T \equiv \frac{-v_{w}}{v_{w w}}=\frac{-1}{\lambda^{i}} \frac{u_{c}^{i}}{v_{w w}}=\frac{-u_{c}^{i}}{u_{c c}^{i} c_{w}^{i}} \Rightarrow c_{w}^{i}=\frac{-u_{c}^{i} / u_{c c}^{i}}{-v_{w} / v_{w w}}=\frac{T^{i}}{T}
$$

which is (7).

Derivation of eqs. (9), (10): Using $p^{i}=T^{i} / T=c_{w}^{i}$ we have

$$
p_{w}^{i}=\frac{T_{w}^{i}}{T}-\frac{T^{i}}{T} \frac{T_{w}}{T}=\frac{1}{T}\left(T_{c}^{i} c_{w}^{i}-\frac{T^{i}}{T} T_{w}\right)=\frac{T^{i}}{T^{2}}\left(T_{c}^{i}-T_{w}\right)=\frac{p^{i}}{T}\left(T_{c}^{i}-T_{w}\right),
$$

which is 97 . In the CRRA settings, $T^{i}=\frac{c^{i}}{\gamma^{i}} \Rightarrow T_{c}^{i}=\frac{1}{\gamma^{i}}$, and

$$
T_{w}=\frac{\partial}{\partial_{w}} \sum_{i} T^{i}=\sum_{i} T_{c}^{i} c_{w}^{i}=E_{\left\{p^{i}\right\}}\left[T_{c}^{i}\right]=E_{\left\{p^{i}\right\}}\left[\frac{1}{\gamma^{i}}\right]
$$

now eq. (9) becomes 10 .

Derivation of eqs. 12), (14): Taking the partial derivative $\frac{\partial}{\partial w}$ of risk aversion $R=\sum_{i} \frac{\gamma^{i} T^{i}}{T}$

$$
R_{w}=\frac{1}{T}\left[\sum_{i} \gamma^{i} T_{c}^{i} c_{w}^{i}-\left(\sum_{i} \gamma^{i} \frac{T^{i}}{T}\right)\left(\sum_{i} T_{c}^{i} c_{w}^{i}\right)\right]=\frac{1}{T} \operatorname{Cov}_{\left\{p^{i}\right\}}\left(\gamma^{i}, T_{c}^{i}\right)=\frac{1}{T} \operatorname{Cov}_{\left\{p^{i}\right\}}\left(\gamma^{i}, \frac{1}{\gamma^{i}}\right)
$$

where we have used (7) $c_{w}^{i}=\frac{T^{i}}{T} \equiv p^{i}$, and in the last equality CRRA utility's property $T_{c}^{i}=\frac{\partial T^{i}}{\partial c^{i}}=$ $\frac{1}{\gamma^{i}}$. 
Taking Ito differential on both sides of $\delta=\sum_{i} \frac{T^{i} \delta^{i}}{T}$, then identifying diffusion and drift parts gives

$$
\begin{aligned}
\delta_{w} & =\frac{1}{T} \sum_{i}\left(\delta^{i}-\delta\right) T_{c}^{i} c_{w}^{i}=\frac{1}{T} \sum_{i}\left(\delta^{i}-\delta\right) T_{c}^{i} \frac{T^{i}}{T}=\frac{1}{T} \operatorname{Cov}_{\left\{p^{i}\right\}}\left(T_{c}^{i}, \delta^{i}\right) \\
\delta_{t} & =\frac{1}{T} \sum_{i}\left(\delta^{i}-\delta\right) T_{c}^{i} c_{t}^{i}=\frac{-1}{T} \sum_{i}\left(\delta^{i}-\delta\right)^{2} T_{c}^{i} T^{i}=\frac{-1}{T} \sum_{i} \frac{\left(\delta^{i}-\delta\right)^{2}}{\gamma^{i}} T^{i}
\end{aligned}
$$

where again the last equality holds for CRRA utilities: $T_{c}^{i}=\frac{1}{\gamma^{i}}$. These concise expressions capture and generalize key results on the behaviors of social discount rate first obtained in Gollier and Zeckhauser (2005) to stochastic environments.

Precautionary savings (prudence) $P \equiv \frac{-w v_{w w w}}{v_{w w}}$, temperance $Q \equiv \frac{-w v_{w w w w}}{v_{w w w}}$ and their relations: Taking the partial derivative $\frac{\partial}{\partial w}$ of risk tolerance $T=\frac{-v_{w}}{v_{w w}}$

$$
T_{w}=-1+\frac{v_{w} v_{w w w}}{v_{w w}^{2}}=-1+\frac{-v_{w}}{w v_{w w}} \frac{-w v_{w w w}}{v_{w w}}=-1+\frac{P}{R} \Rightarrow P=\left(1+T_{w}\right) R=\frac{\left(1+T_{w}\right) w}{T} .
$$

Similarly, since $R=\frac{w}{T}$, and using above expression for $P$ yields a general relation for any time separable utilities (possibly non CRRA)

$$
R_{w}=\frac{1}{T}\left(1-\frac{w T_{w}}{T}\right)=\frac{R}{w}(1+R-P)
$$

which together with (40) implies (17), (41). Combining (63), (17), we have in CRRA setting

$$
R_{w}=\frac{R}{w}(1+R-P)=\frac{1}{T} \operatorname{Cov}_{\left\{p^{i}\right\}}\left(\gamma^{i}, T_{c}^{i}\right)
$$

Very similar to (66), we also have in the general case

$$
P_{w}=\frac{\partial}{\partial w} \frac{-w v_{w w w}}{v_{w w}}=\frac{-v_{w w w}}{v_{w w}}+\frac{w v_{w w w}^{2}}{v_{w w}^{2}}-\frac{w v_{w w w w}}{v_{w w}}=\frac{P}{w}(1+P-Q) .
$$

Next, taking one more time the partial derivative on $T_{w}$ in 65

$$
T_{w w}=\frac{v_{w w} v_{w w w}}{v_{w w}^{2}}+\frac{v_{w} v_{w w w w}}{v_{w w}^{2}}-2 \frac{v_{w} v_{w w w}^{2}}{v_{w w}^{3}}=\frac{P}{w} \frac{(2 P-R-Q)}{R} .
$$


Plugging $R_{w}(66)$ and $P_{w}(68)$ into $\sigma_{\Gamma}^{r}$ in 29$)$ we obtain

$$
\sigma_{\Gamma}^{r}=-\sigma^{w} R\left[\mu^{w}(P-R-1)+\left(\sigma^{w}\right)^{2} P\left(1+\frac{R-Q}{2}\right)\right]
$$

which proves $(30)$.

Derivation of eq. (15), 21): The derivation of the key aggregate relation (65) $P=R+R T_{w}$ must also hold at individual leve ${ }^{36} P^{i}=R^{i}+R^{i} T_{c}^{i}$. Computing the latter's mean in risk-tolerance measure (that is, $E_{\left\{p^{i}\right\}}[X]=\sum_{i} \frac{T^{i}}{T} X^{i}$ ), and taking the difference with the former

$$
\begin{aligned}
P & =E_{\left\{p^{i}\right\}}\left[P^{i}\right]+R T_{w}-E_{\left\{p^{i}\right\}}\left[R^{i} T_{c}^{i}\right]=E_{\left\{p^{i}\right\}}\left[P^{i}\right]+R \sum_{i} T_{c}^{i} c_{w}^{i}-E_{\left\{p^{i}\right\}}\left[R^{i} T_{c}^{i}\right] \\
& =E_{\left\{p^{i}\right\}}\left[P^{i}\right]+E_{\left\{p^{i}\right\}}\left[R^{i}\right] E_{\left\{p^{i}\right\}}\left[T_{c}^{i}\right]-E_{\left\{p^{i}\right\}}\left[R^{i} T_{c}^{i}\right]=E_{\left\{p^{i}\right\}}\left[P^{i}\right]-\operatorname{Cov}_{\left\{p^{i}\right\}}\left(R^{i}, T_{c}^{i}\right) \\
& =E_{\left\{p^{i}\right\}}\left[P^{i}\right]-\operatorname{Cov}_{\left\{p^{i}\right\}}\left(R^{i}, \frac{1}{R^{i}}-\frac{c^{i} R_{c}^{i}}{\left(R^{i}\right)^{2}}\right)
\end{aligned}
$$

where in the last equality we have used $T^{i}=\frac{c^{i}}{R^{i}}$. This is 15 . In the special case when all agents have CRRA utilities, $R^{i}=\gamma^{i}, R_{c}^{i}=0 \forall i, P^{i}=\gamma^{i}+1$, the market-revealed prudence is simplified to

$$
P=E_{\left\{p^{i}\right\}}\left[P^{i}\right]-\operatorname{Cov}_{\left\{p^{i}\right\}}\left(\gamma^{i}, \frac{1}{\gamma^{i}}\right)=E_{\left\{p^{i}\right\}}\left[\gamma^{i}\right]\left(1+E_{\left\{p^{i}\right\}}\left[\frac{1}{\gamma^{i}}\right]\right) \geq E_{\left\{p^{i}\right\}}\left[P^{i}\right]
$$

Same technique can be used on temperances (see 69 ) $Q=2 P-R-w T_{w w} \frac{R}{P}$ and $Q^{i}=2 P^{i}-R^{i}-$ $c^{i} T_{c c}^{i} \frac{R^{i}}{P^{i}}$

$$
Q=E_{\left\{p^{i}\right\}}\left[Q^{i}\right]+2\left(P-E_{\left\{p^{i}\right\}}\left[P^{i}\right]\right)-w T_{w w} \frac{R}{P}+E_{\left\{p^{i}\right\}}\left[c^{i} T_{c c}^{i} \frac{R^{i}}{P^{i}}\right] .
$$

First note that we can derive an agent-based sufficient condition for the convexity of market-revealed

\footnotetext{
${ }^{36}$ We can obtain result at individual level from aggregate result in economy with only a single agent.
} 
precautionary savings

$$
\begin{aligned}
T_{w}=\sum_{i} T_{c}^{i} c_{w}^{i} \Rightarrow T_{w w} & =\sum_{i} T_{c c}^{i}\left(c_{w}^{i}\right)^{2}+\sum_{i} T_{c}^{i} c_{w w}^{i} \\
& =\sum_{i} T_{c c}^{i}\left(c_{w}^{i}\right)^{2}+\sum_{i} T_{c}^{i} \frac{\partial}{\partial w}\left(\frac{T^{i}}{T}\right) \\
& =\sum_{i} T_{c c}^{i}\left(c_{w}^{i}\right)^{2}+\frac{1}{T}\left[\sum_{i}\left(T_{c}^{i}\right)^{2} \frac{T^{i}}{T}-\left(\sum_{i} T_{c}^{i} \frac{T^{i}}{T}\right)^{2}\right] \\
& =\sum_{i} T_{c c}^{i}\left(c_{w}^{i}\right)^{2}+\frac{1}{T} \operatorname{Var}_{\left\{p^{i}\right\}}\left(T_{c}^{i}\right) .
\end{aligned}
$$

Consequently, when $T_{c c}^{i} \geq 0 \forall i$, we also have $T_{w w} \geq 0$. This aggregation property echoes a similar result of proposition 6 . Now plugging $T_{w w}$ into above $Q$, we have

$$
\begin{aligned}
Q & =E_{\left\{p^{i}\right\}}\left[Q^{i}\right]+2\left(P-E_{\left\{p^{i}\right\}}\left[P^{i}\right]\right)-\frac{w R}{P} \sum_{i} T_{c c}^{i} \frac{\left(T^{i}\right)^{2}}{T^{2}}-\frac{w R}{P T} \operatorname{Var}_{\left\{p^{i}\right\}}\left(T_{c}^{i}\right)+E_{\left\{p^{i}\right\}}\left[c^{i} T_{c c}^{i} \frac{R^{i}}{P^{i}}\right] \\
& =E_{\left\{p^{i}\right\}}\left[Q^{i}\right]+2\left(P-E_{\left\{p^{i}\right\}}\left[P^{i}\right]\right)-\frac{w R}{P T} \operatorname{Var}_{\left\{p^{i}\right\}}\left(T_{c}^{i}\right)+E_{\left\{p^{i}\right\}}\left[T^{i} T_{c c}^{i}\left(\frac{\left(R^{i}\right)^{2}}{P^{i}}-\frac{R^{2}}{P}\right)\right] \\
& =E_{\left\{p^{i}\right\}}\left[Q^{i}\right]-2 \operatorname{Cov}_{\left\{p^{i}\right\}}\left(R^{i}, \frac{1}{R^{i}}-\frac{c^{i} R_{c}^{i}}{\left(R^{i}\right)^{2}}\right)-\frac{R^{2}}{P} \operatorname{Var}_{\left\{p^{i}\right\}}\left(T_{c}^{i}\right)+E_{\left\{p^{i}\right\}}\left[T^{i} T_{c c}^{i}\left(\frac{\left(R^{i}\right)^{2}}{P^{i}}-\frac{R^{2}}{P}\right)\right] .
\end{aligned}
$$

In the special case when all agents have CRRA utilities, $R^{i}=\gamma^{i}, R_{c}^{i}=0, T_{c c}^{i}=0 \forall i$, the marketrevealed temperance is simplified to (21)

$$
Q=E_{\left\{p^{i}\right\}}\left[Q^{i}\right]-2 \operatorname{Cov}_{\left\{p^{i}\right\}}\left(\gamma^{i}, \frac{1}{\gamma^{i}}\right)-\frac{R^{2}}{P} \operatorname{Var}_{\left\{p^{i}\right\}}\left(\frac{1}{\gamma^{i}}\right)
$$

Derivation of Hansen-Jagannathan bound (26): Let $S(w, t)$ be price of the contingent claim (i.e., stock) on the dividend stream,

$S(w, t)=E_{t}\left[\frac{M(t+d t)}{M(t)}\{S(w+d w, t+d t)+d w\}\right] \Rightarrow E_{t}\left[\frac{M(t+d t)}{M(t)} \frac{S(w+d w, t+d t)+d w}{S(w, t)}\right]=1$.

Next, since $M(t+d t)=M(t)[1-r(w, t) d t-\eta(w, t) d Z(t)]$, up to order $d t$ we have

$$
E_{t}\left[\frac{M(t+d t)}{M(t)}\{1+r(w, t) d t\}\right]=1
$$


Combining these identities yields

$$
E_{t}\left[\frac{M(t+d t)}{M(t)}\left(\frac{S(w+d w, t+d t)+d w}{S(w, t)}-1-r(w, t) d t\right)\right]=0
$$

where $\frac{S(w+d w, t+d t)+d w}{S(w, t)}-1-r(w, t) d t$ is simply the stock excess return. Standard argument that the absolute value of correlation between this and the stochastic discount factor $\frac{M(t+d t)}{M(t)}$ is less than unity implies (after plugging in (i) the mean value $1-r d t$ and standard deviation $\eta \sqrt{d t}$ of $\frac{M(t+d t)}{M(t)}$, (ii) the expected stock excess return $E_{t}\left[\frac{S(w+d w, t+d t)+d w}{S(w, t)}-1-r(w, t) d t\right]=\left(\mu^{s}-r\right) d t$ by virtue of gain, and (iii) the notation $\sigma_{\mu^{s}-r} d t$ for stock excess return volatility)

$$
\eta \sqrt{d t} \geq[1-r(w, t) d t] \frac{\left|\mu^{s}(w, t)-r(w, t)\right| d t}{\sigma_{\mu^{s}-r} d t}
$$

Finally, to use annual data, we somewhat coarsely set $d t=1$. Since the expected stock excess return is positive, this is precisely the bound (26).

\section{A.2 Proofs of propositions}

Proof of proposition 1. Market-revealed risk tolerance: since $\sum_{i} c^{i}=w \rightarrow \sum_{i} c_{w}^{i}=1$,

$$
\sum_{i} T^{i}=T \quad \text { or } \quad \sum_{i} p^{i} \equiv \sum_{i} \frac{T^{i}}{T}=1
$$

Market-revealed risk aversion

$$
R \equiv-w \frac{v_{w w}}{v_{w}}=-w \frac{\frac{1}{\lambda^{i}} u_{c c}^{i} c_{w}^{i}}{\frac{1}{\lambda^{i}} u_{c}^{i}}=-w \frac{u_{c c}^{i}}{u_{c}^{i}} \frac{T^{i}}{T}=\sum_{i} \frac{-c^{i} u_{c c}^{i}}{u_{c}^{i}} \frac{T^{i}}{T}=\sum_{i} \frac{T^{i} R^{i}}{T} .
$$

Market-revealed discount factor

$$
\delta \equiv-\frac{v_{w t}}{v_{w}}=-\frac{u_{c t}^{i}+u_{c c}^{i} c_{t}^{i}}{u_{c}^{i}}=\sum_{i} \frac{T^{i}}{T}\left(\frac{-u_{c t}^{i}}{u_{c}^{i}}+\frac{c_{t}^{i}}{T^{i}}\right)=\sum_{i} \frac{T^{i} \delta^{i}}{T}+\frac{\sum_{i} c_{t}^{i}}{T}=\sum_{i} \frac{T^{i} \delta^{i}}{T},
$$

because $\sum_{i} c_{t}^{i}=\frac{\partial\left(\sum_{i} c^{i}\right)}{\partial t}=\frac{\partial w}{\partial t}=0$ as aggregate endowment $w$ and time $t$ are two independent variables. 


\section{Proof of proposition 2 ,}

$$
\begin{aligned}
& \frac{\partial E_{\left\{p^{i}\right\}}\left[a^{i}\right]}{\partial w}=\frac{\partial}{\partial w} \sum_{i} a^{i} p^{i}=\sum_{i} a_{w}^{i} p^{i}+\sum_{i} a^{i} p_{w}^{i} \\
& =\sum_{i} a_{w}^{i} p^{i}+\sum_{i} a^{i} \frac{p_{w}^{i}}{p^{i}} p^{i}-\sum_{i} a^{i} p^{i} \sum_{j} \frac{p_{w}^{j}}{p^{j}} p^{j}+\sum_{i} a^{i} p^{i} \sum_{j} \frac{p_{w}^{j}}{p^{j}} p^{j} \\
& =E_{\left\{p^{i}\right\}}\left[a_{w}^{i}\right]+\operatorname{Cov}_{\left\{p^{i}\right\}}\left(a^{i}, \frac{p_{w}^{i}}{p^{i}}\right)+\sum_{i} a^{i} p^{i} \sum_{j} \frac{p_{w}^{j}}{p^{j}} p^{j} \\
& =E_{\left\{p^{i}\right\}}\left[a_{w}^{i}\right]+\operatorname{Cov}_{\left\{p^{i}\right\}}\left(a^{i}, \frac{p_{w}^{i}}{p^{i}}\right)+E_{\left\{p^{i}\right\}}\left[a^{i}\right] \frac{\partial}{\partial w} \sum_{j} p^{j}=E_{\left\{p^{i}\right\}}\left[a_{w}^{i}\right]+\operatorname{Cov}_{\left\{p^{i}\right\}}\left(a^{i}, \frac{p_{w}^{i}}{p^{i}}\right) .
\end{aligned}
$$

The last equality holds because $\sum_{j} p^{j}=1$, and hence term $E_{\left\{p^{i}\right\}}\left[a^{i}\right] \frac{\partial}{\partial w} \sum_{j} p^{j}=0$.

Proof of proposition 3 . For CRRA utilities, eq. (71) shows that market-revealed prudence $P$ is always larger or equal average prudence $E_{\left\{p^{i}\right\}}\left[P^{i}\right]$ under risk tolerance measure $\left\{p^{i}=\frac{T^{i}}{T}\right.$. In the case of 2-CRRA economy $(i=A, B)$ (and assume without loss of generality throughout that $\gamma^{A}<\gamma^{B}$ ), plugging $P^{i}=\gamma^{i}+1$ into 71

$$
\begin{aligned}
P & =E_{\left\{p^{i}\right\}}\left[\gamma^{i}+1\right]-1+E_{\left\{p^{i}\right\}}\left[\gamma^{i}\right] E_{\left\{p^{i}\right\}}\left[\frac{1}{\gamma^{i}}\right]=E_{\left\{p^{i}\right\}}\left[\gamma^{i}\right]\left(1+E_{\left\{p^{i}\right\}}\left[\frac{1}{\gamma^{i}}\right]\right) \\
& =\left(p^{A} \gamma^{A}+\left(1-p^{A}\right) \gamma^{B}\right)\left(1+\frac{p^{A}}{\gamma^{A}}+\frac{1-p^{A}}{\gamma^{B}}\right) .
\end{aligned}
$$

Precautionary savings $P$ is an explicit concave quadratic function of $p^{A}$. Theoretically ${ }^{37}$ it obtains maximum value

$$
P^{*} \equiv \max P=\frac{\left(\gamma^{A}+\gamma^{B}+\gamma^{A} \gamma^{B}\right)^{2}}{4 \gamma^{A} \gamma^{B}} \quad \text { at } \quad p^{A *}=\frac{1}{2}+\frac{1}{2} \frac{\gamma^{A} \gamma^{B}}{\gamma^{A}-\gamma^{B}}
$$

Evidently, when $\frac{\gamma^{B}}{\gamma^{B}+1} \geq \gamma^{A}, p^{A *} \in[0,1]$ and the above value $P^{*}$ is indeed market-revealed prudence's legitimate maximum. Furthermore in this case, market-revealed prudence $P\left(p^{A}\right)$ is larger than the largest individual prudence (which is agent $B$ 's under current convention) $P^{B}=\gamma^{B}+1$ for all $0 \leq p^{A} \leq 2 p^{A *}=1+\frac{\gamma^{A} \gamma^{B}}{\gamma^{A}-\gamma^{B}}$. However, when $\frac{\gamma^{B}}{\gamma^{B}+1}<\gamma^{A}, p^{A *}<0$, the market-revealed prudence's legitimate maximum is $P^{*}=P^{B}=\gamma^{B}+1$, which is attained at $p^{A *}=0$.

\footnotetext{
${ }^{37}$ This is indeed the legitimate maximum when the corresponding $\operatorname{argmax} p^{A *} \in[0,1]$.
} 
Proof of proposition 4. For CRRA utilities $\left(Q^{i}=\gamma^{i}+2\right)$, from eqs. (72) and 71 )

$$
\begin{aligned}
Q & =R+2 R E_{\left\{p^{i}\right\}}\left[\frac{1}{\gamma^{i}}\right]-\frac{R^{2}}{P}\left(E_{\left\{p^{i}\right\}}\left[\frac{1}{\left(\gamma^{i}\right)^{2}}\right]-\left(E_{\left\{p^{i}\right\}}\left[\frac{1}{\gamma^{i}}\right]\right)^{2}\right) \\
& =R\left(1+2 E_{\left\{p^{i}\right\}}\left[\frac{1}{\gamma^{i}}\right]+\frac{\left(E_{\left\{p^{i}\right\}}\left[\frac{1}{\gamma^{i}}\right]\right)^{2}-E_{\left\{p^{i}\right\}}\left[\frac{1}{\left(\gamma^{i}\right)^{2}}\right]}{1+E_{\left\{p^{i}\right\}}\left[\frac{1}{\gamma^{i}}\right]}\right) \\
& =E_{\left\{p^{i}\right\}}\left[\gamma^{i}\right]\left(3 E_{\left\{p^{i}\right\}}\left[\frac{1}{\gamma^{i}}\right]+\frac{1-E_{\left\{p^{i}\right\}}\left[\frac{1}{\left(\gamma^{i}\right)^{2}}\right]}{1+E_{\left\{p^{i}\right\}}\left[\frac{1}{\gamma^{i}}\right]}\right) .
\end{aligned}
$$

Next, using 68 $P_{w}=\frac{P(1+P-Q)}{w}$ we see that $Q>P+1$ if and only if $P_{w} \leq 0$. Specializing in the 2-CRRA economy, we have

$$
P_{w}=\frac{\partial P}{\partial p^{A}} \frac{\partial p^{A}}{\partial w}=\left(\gamma^{A}-\gamma^{B}\right)\left(1+\frac{p^{A}-p^{B}}{\gamma^{A}}+\frac{p^{B}-p^{A}}{\gamma^{B}}\right) \frac{T^{A} T^{B}}{T^{3}} \frac{\gamma^{B}-\gamma^{A}}{\gamma^{A} \gamma^{B}}
$$

where we have used the explicit expressions for $P\left(73\right.$ and $p_{w}^{A} 62$. It is now clear that $P_{w} \leq 0$, or equivalently $Q>P+1$, if and only if (note that $p^{A}+p^{B}=1$ and we have assumed $\gamma^{A}<\gamma^{B}$ throughout)

$$
1+\frac{p^{A}-p^{B}}{\gamma^{A}}+\frac{p^{B}-p^{A}}{\gamma^{B}} \geq 0 \Leftrightarrow p^{A} \geq p^{A *} \equiv \frac{1}{2}+\frac{1}{2} \frac{\gamma^{A} \gamma^{B}}{\gamma^{A}-\gamma^{B}} .
$$

We note that when $\frac{\gamma^{B}}{\gamma^{B}+1}<\gamma^{A}, p^{A *}<0$. In this case we simply have $Q>P+1$ for all $p^{A}>0$. The value $p^{A *} \equiv \frac{1}{2}+\frac{1}{2} \frac{\gamma^{A} \gamma^{B}}{\gamma^{A}-\gamma^{B}}$ is also where the market-revealed precautionary savings $P$ attains maximum (see (74)).

Proof of proposition 5. This proposition holds on the premise of the large precautionary savings $P>\frac{2 \mu^{w}}{\left(\sigma^{w}\right)^{2}} 25$ needed for the observed low real interest rate.

Case $Q>\frac{2 \mu^{w}}{\left(\sigma^{w}\right)^{2}}+R+2$ : we first rewrite 30 as

$$
\sigma_{\Gamma}^{r}=\mu^{w} \sigma^{w} R\left(\left[\frac{\left(\sigma^{w}\right)^{2}}{2 \mu^{w}}(Q-R-2)-1\right] P+R+1\right) .
$$

Since the expression inside square brackets is positive in the current case, large precautionary 
savings 25] implies

$$
\begin{aligned}
\sigma_{\Gamma}^{r} & >\mu^{w} \sigma^{w} R\left(\left[\frac{\left(\sigma^{w}\right)^{2}}{2 \mu^{w}}(Q-R-2)-1\right] \frac{2 \mu^{w}}{\left(\sigma^{w}\right)^{2}}+R+1\right) \\
& =\mu^{w} \sigma^{w} R\left(Q-1-\frac{2 \mu^{w}}{\left(\sigma^{w}\right)^{2}}\right) \approx \mu^{w} \sigma^{w} R\left(Q-\frac{2 \mu^{w}}{\left(\sigma^{w}\right)^{2}}\right),
\end{aligned}
$$

which is 32 (the last approximation is from the conditions $Q-\frac{2 \mu^{w}}{\left(\sigma^{w}\right)^{2}}>R+2$ and bound 27 ) ${ }^{38}$ Case $Q<\frac{2 \mu^{w}}{\left(\sigma^{w}\right)^{2}}$ : we first rewrite 30 as

$$
\sigma_{\Gamma}^{r}=\sigma^{w} R \frac{\left(\sigma^{w}\right)^{2}}{2}\left(P\left[Q-\frac{2 \mu^{w}}{\left(\sigma^{w}\right)^{2}}\right]+R\left[\frac{2 \mu^{w}}{\left(\sigma^{w}\right)^{2}}-P\right]+\left[\frac{2 \mu^{w}}{\left(\sigma^{w}\right)^{2}}-2 P\right]\right)
$$

In the current case, all three expressions inside square brackets are negative under large precautionary savings condition 25$]$, and thus

$$
\sigma_{\Gamma}^{r}<\sigma^{w} R \frac{\left(\sigma^{w}\right)^{2}}{2} P\left[Q-\frac{2 \mu^{w}}{\left(\sigma^{w}\right)^{2}}\right]<\mu^{u} \sigma^{w} R\left[Q-\frac{2 \mu^{w}}{\left(\sigma^{w}\right)^{2}}\right]
$$

which is (33) (the last inequality is again from the conditions (25)).

Proof of proposition 6. First we note from 65 that $P=\left(1+T_{w}\right) R$, which implies

$$
P \geq R+1 \Leftrightarrow T_{w} \geq \frac{1}{R}=\frac{T}{w} \Leftrightarrow w T_{w} \geq T ; \quad \text { similarly } P^{i} \geq R^{i}+1 \Leftrightarrow c^{i} T_{c}^{i} \geq T^{i} .
$$

Next, since $T=\sum_{i} T^{i}$ and $c_{w}^{i}=\frac{T^{i}}{T}$

$w T_{w}-T=w \sum_{i} T_{c}^{i} c_{w}^{i}-T=\frac{\left(\sum_{i} c^{i}\right)\left(\sum_{i} T_{c}^{i} T^{i}\right)-T^{2}}{T} \geq \frac{\left(\sum_{i} \sqrt{c^{i} T_{c}^{i} T^{i}}\right)^{2}-T^{2}}{T} \geq \frac{\left(\sum_{i} T^{i}\right)^{2}-T^{2}}{T}=0$

where the first inequality is an application of Cauchy-Schwarz's, the second arises from the proposition's hypothesis (75). Now $w T_{w}-T \geq 0$ is equivalent to $P \geq R+1$ again by virtue of (75).

\footnotetext{
${ }^{38}$ In the same approximation, in the statement of proposition 5 we write $Q>\frac{2 \mu^{w}}{\left(\sigma^{w}\right)^{2}}+R$ in place of $Q>\frac{2 \mu^{w}}{\left(\sigma^{w}\right)^{2}}+R+2$. Practically, the difference is non-material by virtue of empirically large value $\frac{2 \mu^{w}}{\left(\sigma^{w}\right)^{2}} \sim 100$.
} 


\section{B Proofs concerning asset return volatilities}

\section{Preliminaries:}

When $\theta$ is a continuously differentiable function of the underlying Brownian motion $Z$, the Malliavin derivative $\mathcal{D}_{t} \theta$ is the deviation in $\theta$ due to change in the path of $Z$ starting at $t$. The Malliavin calculus is a handy tool to study stock return volatilities. We adopt this tool here along the presentation of Detemple et. al. (2003) and Bhamra and Uppal (2009). More extensive exposition of this powerful tool can be found in Nualart (2006). We first state two useful results for our proofs.

Result 1: Let $\beta(t)$ be a general GBM process with bounded drift and diffusion

$$
\frac{d \beta(t)}{\beta(t)}=\mu(\beta, t) d t+\sigma(\beta, t) d Z(t) \quad \text { where }|\mu(\beta, t)|,|\sigma(\beta, t)|<\infty \quad \text { almost surely. }
$$

Then the process $\beta(t)$ never changes its sign

$$
\beta(t) \beta(s) \geq 0 \quad \forall t, s \quad \text { almost surely. }
$$

Result 2: Let $\theta(t)$ be a general diffusion process

$$
d \theta(t)=\mu(\theta, t) d t+\sigma(\theta, t) d Z(t)
$$

then under regularity conditions the Malliavin derivative $\Theta(\tau) \equiv \mathcal{D}_{t} \theta(\tau)$ of process $\theta(t)$ is a generalized GBM process with specified initial value

$$
\frac{d \Theta(\tau)}{\Theta(\tau)}=\mu_{\theta}(\theta, \tau) d \tau+\sigma_{\theta}(\theta, \tau) d Z(\tau) ; \quad \Theta(t)=\sigma(\theta, t)
$$

Note that subscript $\theta$ in $\mu_{\theta}, \sigma_{\theta}$ always denotes the partial derivative and Malliavin derivative $\mathcal{D}_{t} \theta(\tau)$ is a process with respect to the ulterior time $\tau$, and thus is defined only for $\tau \geq t$. This result makes clear the relation between diffusion of a process and its Malliavin derivative. More specifically,

$$
\mathcal{D}_{t} \theta(\tau)=\Theta(\tau)=\sigma(\theta, t) \exp \left\{\int_{t}^{\tau}\left(\mu_{\theta}(\theta, u)-\frac{1}{2} \sigma_{\theta}^{2}(\theta, u)\right) d u+\int_{t}^{\tau} \sigma_{\theta}(\theta, u) d Z(u)\right\}
$$

In particular they are identical when the Malliavin derivative is contemporaneous, $\mathcal{D}_{t} \theta(t)=\sigma(\theta, t)$. 
In case of two-CRRA-agent economies, working with first agent's risk tolerance measure $p^{A}$ is also convenient for our technical proofs. Applying Ito lemma on $p^{A}=\frac{T^{A}}{T}$ yields the dynamics of this state variable Indeed, the general volatility $\sigma^{p A}$ and drift $\mu^{p A}$ of this state variable's diffusion process

$$
\begin{gathered}
\frac{d p^{A}(w, t)}{p^{A}(w, t)}=\mu^{p A}\left(p^{A}\right) d t+\sigma^{p A}\left(p^{A}\right) d Z(t), \\
\sigma^{p A}\left(p^{A}\right)=\sigma^{w} R p^{B}\left(\frac{1}{\gamma^{A}}-\frac{1}{\gamma^{B}}\right), \\
\mu^{p A}\left(p^{A}\right)=p^{B}\left[-\frac{R\left(\delta^{A}-\delta^{B}\right)}{\gamma^{A} \gamma^{B}}+R \mu^{w}\left(\frac{1}{\gamma^{A}}-\frac{1}{\gamma^{B}}\right)+\left(\sigma^{w}\right)^{2}\left(\frac{1}{\gamma^{A}}-\frac{1}{\gamma^{B}}\right)\left(\frac{R}{2 \gamma^{A} \gamma^{B}}-\frac{p^{A}}{\gamma^{A}}-\frac{p^{B}}{\gamma^{B}}\right)\right] .
\end{gathered}
$$

where $p^{B}=1-p^{A}$, and $R\left(p^{A}\right)=p^{A} \gamma^{A}+p^{B} \gamma^{B}$ is the aggregate risk aversion in (6). We now proceed to the proofs.

Derivation of mpr volatility 41): plugging $R_{w}$ in (17) into (40), we immediately obtain (41).

Derivation of eq. (38): Taking the Malliavin derivative $\mathcal{D}_{t}$ in measure $\mathcal{Q}$ of both sides of eq. (36) yields

$$
\begin{gathered}
\sigma^{s \mathcal{Q}}(w, t) S(w, t) e^{-\int_{0}^{t} r(s) d s}=E_{t}^{\mathcal{Q}}\left[\mathcal{D}_{t} G(t, T)\right], \\
G(t, T) \equiv \int_{t}^{T} e^{-\int_{0}^{u} r(\tau) d \tau} w(u) d u
\end{gathered}
$$

where $\sigma^{s \mathcal{Q}}$ is the stock return volatility in measure $\mathcal{Q}$. The diffusion invariance principle $\sigma^{s \mathcal{Q}}=\sigma^{s}$ justifies the drop of superscript $\mathcal{Q}$ hereafter. Using the explicit aggregate endowment process (1) in measure $\mathcal{Q}$

$$
w(t)=w(0) \exp \left[\left(\mu^{w}-\frac{\left(\sigma^{w}\right)^{2}}{2}\right) t+\sigma^{w} Z^{\mathcal{Q}}(t)-\sigma^{w} \int_{0}^{t} \eta(w, u) d u\right]
$$

and the chain rule we obtain Malliavin derivative

$$
\mathcal{D}_{t} G(t, T)=\int_{t}^{T} d u w(u) e^{-\int_{0}^{u} r(\tau) d \tau}\left\{\sigma^{w}-\sigma^{w} \int_{t}^{u} d \tau \mathcal{D}_{t} \eta(w, \tau)-\int_{t}^{u} d \tau \mathcal{D}_{t} r(w, \tau)\right\}
$$

Plugging above $\mathcal{D}_{t} G(t, T)$ into eq. 82 we get the excess volatility of stock return (38). 
Derivation of eq. (39): Let's define

$$
\theta(w, t) \equiv \sigma^{w} \eta(w, t)+r(w, t) ; \quad d \theta=\mu^{\theta} d t+\sigma^{\theta} d Z(t)
$$

From (38), it is clear that $\mathcal{D}_{t} \theta(w, \tau)<0 \forall \tau \geq t$ implies positive stock return excess volatility $\sigma^{s}>\sigma^{w}$. In light of Result 2 above, this Malliavin derivative is a generalized Brownian motion, and Result 1 implies that it will remain negative at all time if all following conditions hold.

1. Diffusion $\sigma_{\theta}^{\theta} \equiv \frac{\partial \sigma^{\theta}}{\partial \theta}$ is bounded. Indeed this is the case. In the current two-CRRA-agent setting, $\delta, R, P$ are simple polynomials of $p^{A}$, and so are $r, \eta$ in (24), and also $\theta$ and $\sigma^{\theta}=$ $\left[\partial\left(r+\sigma^{w} \eta\right) / \partial p^{A}\right] \sigma^{p A}$ by virtue of 81 . Then the next-generation partial derivatives $\theta_{p^{A}} \equiv \frac{\partial \theta}{\partial p^{A}}$ and $\sigma_{p^{A}}^{\theta} \equiv \frac{\partial \sigma^{\theta}}{\partial p^{A}}$ are also simple polynomials of $p^{A}$. These in turn imply $\sigma_{\theta}^{\theta}=\frac{\partial \sigma^{\theta}}{\partial \theta}=\frac{\sigma_{p^{A}}^{\theta}}{\theta_{p^{A}}}$ is bounded almost surely because $p^{A}$ is in $(0,1)$.

2. Drift $\mu_{\theta}^{\theta} \equiv \frac{\partial \mu^{\theta}}{\partial \theta}$ is bounded. This holds by identical reasoning.

3. Initial value $\left.\mathcal{D}_{t} \theta(w, \tau)\right|_{\tau=t}<0$. Note that because $\tau=t$, this Malliavin derivative is simply the volatility $\sigma^{\theta}=\left[\partial\left(r+\sigma^{w} \eta\right) / \partial p^{A}\right] \sigma^{p A}$. From $\left[81, \sigma^{p A}\right.$ is always positive for our convention $\gamma^{A}<\gamma^{B}$, then this last condition is precisely the required sufficient condition 39.

Derivation of eq. 42): In 2-agent economy, we can work with risk tolerance measure $p^{A} \equiv \frac{T^{A}}{T}$ as key underlying state variable. Using (24)

$$
\begin{aligned}
& \frac{\partial\left(r+\sigma^{w} \eta\right)}{\partial p^{A}}=\frac{1}{p_{w}^{A}}\left(r_{w}+\sigma^{w} \eta_{w}\right)=\frac{1}{p_{w}^{A}}\left[\delta_{w}+\left(\sigma^{w}\right)^{2}\left(\frac{\mu^{w}}{\left(\sigma^{w}\right)^{2}}+1-\frac{P}{2}\right) R_{w}-\frac{1}{2}\left(\sigma^{w}\right)^{2} R P_{w}\right] \\
& =\frac{1}{p_{w}^{A}} \frac{R}{w}\left[\operatorname{Cov}_{\left\{p^{i}\right\}}\left(\delta^{i}, \frac{1}{\gamma^{i}}\right)+\left(\sigma^{w}\right)^{2}\left(\frac{\mu^{w}}{\left(\sigma^{w}\right)^{2}}+1-\frac{P}{2}\right)(R+1-P)-\frac{1}{2}\left(\sigma^{w}\right)^{2} P(1+P-Q)\right] \\
& =\frac{1}{p_{w}^{A}} \frac{R}{w}\left[\operatorname{Cov}_{\left\{p^{i}\right\}}\left(\delta^{i}, \frac{1}{\gamma^{i}}\right)+\left(\sigma^{w}\right)^{2}\left(\left\{\frac{\mu^{w}}{\left(\sigma^{w}\right)^{2}}+1\right\}\{R+1\}-\frac{\mu^{w} P}{\left(\sigma^{w}\right)^{2}}+\frac{P(Q-R-4)}{2}\right)\right] .
\end{aligned}
$$

where the second equality arises from (64), (66), 68). Next, since $\sigma^{p A}=p_{w}^{A} \sigma^{w}$, together with convention $\gamma^{A}<\gamma^{B}$ and 81 , we have $p_{w}^{A}>0$. From 83 , the derivative $\frac{d\left(r+\sigma^{w} \eta\right)}{d p^{A}}$ in 39 is negative only if the expression in square brackets is negative

$$
\left(\sigma^{w}\right)^{2}\left(\left\{\frac{\mu^{w}}{\left(\sigma^{w}\right)^{2}}+1\right\}\{R+1\}-\frac{\mu^{w} P}{\left(\sigma^{w}\right)^{2}}+\frac{P(Q-R-4)}{2}\right)<-\operatorname{Cov}_{\left\{p^{i}\right\}}\left(\delta^{i}, \frac{1}{\gamma^{i}}\right)
$$


For empirically reasonable values of aggregate consumption moments $\mu^{w} \sim 2 \%, \sigma^{w} \sim 2 \%$, we have $\frac{\mu^{w}}{\left(\sigma^{w}\right)^{2}} \geq 1$, above condition becomes 42 . Thus, 42 implies $\sqrt{39}$, so it is also a sufficient condition for positive stock return excess volatility.

\section{Proofs concerning heterogeneity transformations}

Proof of proposition 7. The multiplicative factor $\Upsilon(Z(t), t) 47$ is required to be able to reduce FOC (46) to a simpler FOC 49, thus it satisfies

$$
e^{-\delta^{A} t} \xi^{A}(\Upsilon)^{\gamma^{A}}=e^{-\delta^{B} t} \xi^{B}(\Upsilon)^{\gamma^{B}}
$$

Let us look for $\Upsilon$ in the form $\exp \left(\beta^{\gamma, \delta} t\right) \exp \left(\beta^{\gamma, \theta} Z(t)\right)$. Plugging in the Radon-Nikodym derivative $\xi^{i}=e^{-\left(\theta^{i}\right)^{2} t / 2} e^{-\theta^{i} Z(t)}$, above eq. becomes

$$
\begin{aligned}
& \exp \left[\left(\gamma^{A} \beta^{\gamma, \delta}-\delta^{A}-\frac{\left(\theta^{A}\right)^{2}}{2}\right) t\right] \exp \left[\left(\gamma^{A} \beta^{\gamma, \theta}-\theta^{A}\right) Z(t)\right] \\
= & \exp \left[\left(\gamma^{B} \beta^{\gamma, \delta}-\delta^{B}-\frac{\left(\theta^{B}\right)^{2}}{2}\right) t\right] \exp \left[\left(\gamma^{B} \beta^{\gamma, \theta}-\theta^{B}\right) Z(t)\right] .
\end{aligned}
$$

Identifying the drift and diffusion parts immediately yields $\beta^{\gamma, \delta}, \beta^{\gamma, \theta}$ in 48 . This transformation implements the isomorphism $\left\{\gamma^{1}, \gamma^{2}, \delta^{1}, \delta^{2}, \theta^{1}, \theta^{2}, w(t)\right\} \longleftrightarrow\left\{\gamma^{1}, \gamma^{2}, \hat{w}(t)\right\}$. 


\section{References}

[1] Aarbu, K. O. and F. Schroyen (2009), "Mapping risk aversion in Norway using hypothetical income gambles," Working paper, Dept. of Economics, Norwegian School of Economics and Business Administration.

[2] Alan S. and M. Browning (2010), "Estimating Intertemporal Allocation Parameters using Synthetic Residual Estimation," Review of Economic Studies, 77, 12311261.

[3] Bansal, R., and A. Yaron (2004), "Risks for the Long Run: A Potential Resolution of Asset Pricing Puzzles," Journal of Finance, 59, 14811509.

[4] Barsky, R. B., F. T. Juster, M. S. Kimball and M. D. Shapiro (1997), "Preference parameters and behavioral heterogeneity: An experimental approach in the HRS," Quarterly Journal of Economics, 112, S537S579.

[5] Basak, Suleyman (2005), "Asset Pricing with Heterogeneous Beliefs," Journal of Banking and Finance, 29, 2849-2881.

[6] Brennan, M. J. (1998), "The role of learning in dynamic portfolio decisions," European Finance Review, 1, 295-306.

[7] Benninga, S and J. Mayshar (2000), "Heterogeneity and Option Pricing," Review of Derivatives Research, 4, 7-27.

[8] Bhamra, Harjoat S. and Raman Uppal (2009), "The Effect of Introducing a Non-Redundant Derivative on the Volatility of Stock-Market Return When Agents Differ in Risk Aversion," Review of Financial Studies, 22, 2303-2330.

[9] Bhamra, Harjoat S. and Raman Uppal (2010), "Asset Prices with Heterogeneity in Preferences and Beliefs," Working paper, Sauder School of Business (UBC) and London Business School.

[10] Blume, L. and D. Easley (2006) "If you're so smart, why aren't you rich? Belief selection in complete and incomplete markets", Econometrica, 74, 929-966.

[11] Campbell, J. Y. and J. H. Cochrane (1999), "By Force of Habit: A Consumption-Based Explanation of Aggregate Stock Market Behavior," Journal of Political Economy, 107, 205-251. 
[12] Campbell, J. Y. (2003), "Consumption-based Asset Pricing," in Constantinides, G., M. Harris and R. Stulz (eds) Handbook of the Economics of Finance (Amsterdam: North-Holland) 803887.

[13] Chen, H., S. Joslin and N-K. Tran (2010), "Rare Disasters and Risk Sharing with Heterogeneous Beliefs," MIT Sloan School of Management and NBER working paper No 16035.

[14] Chesson, H. and W. K. Viscusi (2000), "The heterogeneity of time-risk tradeoffs," Journal of Behavioral Decision Making, 13, 251-258.

[15] Cox J. C. and C.-F. Huang (1989), "Optimal consumption and portfolio policies when asset prices follow a diffusion process," Journal of Economic Theory, 49, 3383.

[16] Dimson, E., P. Marsh and M. Staunton (2008), "The Worldwide Equity Premium: A Smaller Puzzle," in Mehra R. and E. Prescott (eds) Handbook of the Equity Risk Premium (Elsevier B.V.) 467-514 (also SSRN Working Paper No. 891620).

[17] Detemple, J. B., R. Garcia and M. Rindisbacher (2003), "A Monte Carlo Method for Optimal Portfolios," Journal of Finance, 58, 40146.

[18] Detemple, J. and Murthy, S. (1994), "Intertemporal asset pricing with heterogeneous beliefs", Journal of Economic Theory, 62, 294-320.

[19] Dumas, B. (1989), "Two-Person Dynamic Equilibrium in the Capital Market," Review of Financial Studies, 2, 157188.

[20] Dumas, B., A. Kurshev and R. Uppal (2009) "Equilibrium Portfolio Strategies in the Presence of Sentiment Risk and Excess Volatility," Journal of Finance, 64, 579-629.

[21] Gallmeyer, M. and B. Hollifield (2008) "An Examination of Heterogeneous Beliefs with a Short Sale Constraint," Review of Finance, 12, 323-364.

[22] Garleanu, N. and S. Panageas (2010), "Young, Old, Conservative and Bold: The Implications of Heterogeneity and Finite Lives for Asset Pricing," Working Paper, University of California at Berkeley and University of Chicago.

[23] Gollier, Christian, and Richard Zeckhauser (2005), "Aggregation of Heterogeneous Time Preferences," Journal of Political Economy, 113, 878-96. 
[24] Hansen, L. P., and R. Jagannathan (1991), "Implications of security market data for models of dynamic economies," Journal of Political Economy, 99, 225262.

[25] Jouini, E. and C. Napp, (2007), "Consensus Consumer and Intertemporal Asset Pricing with Heterogeneous Beliefs," Review of Economic Studies, 74, 1149-1174.

[26] Karatzas, I, J. P. Lehoczky, and S. E. Shreve (1987), "Optimal portfolio and consumption decisions for a "small investor" on a finite horizon," SIAM Journal on Control and Optimization, 25,15571586 .

[27] Karatzas, I. and S. E. Shreve (1991), "Brownian Motion and Stochastic Calculus," 2nd ed., Springer, New York.

[28] Kimball, M. S. (1990), "Precautionary Saving in the Small and in the Large," Econometrica, $58,53-73$.

[29] Kimball, M. S. (1992), "Precautionary Motives for Holding Assets," The New Palgrave Dictionary of Money and Finance, Peter Newman, Murray Milgate and John Eatwell (eds.), Stockton Press, New York, 158-161.

[30] Kimball, M. S. (1993), "Standard Risk Aversion," Econometrica, 61, 589-611.

[31] Kimball, M. S., C. R. Sahm and M. D. Shapiro (2008), "Imputing risk tolerance from survey responses," Journal of the American Statistical Association, 103, 10281038.

[32] Kogan, L., S. Ross, J. Wang and M. Westerfield (2006), "The Price Impact and Survival of Irrational Traders," Journal of Finance, 61, 195229.

[33] Kogan, L., S. Ross, J. Wang and M. Westerfield (2009). "Market Selection," Working paper, MIT Sloan School of Management.

[34] Leland, H. E. (1968) "Saving and Uncertainty: The Precautionary Demand for Saving," Quarterly Journal of Economics, 82, 465-473.

[35] Lengwiler, Yvan (2005), "Heterogeneous Patience and the Term Structure of Interest Rates," American Economic Review, 95, 890-896. 
[36] Lengwiler, Yvan, Semyon Malamud and Eugene Trubowitz (2005), "Asset Pricing in Heterogeneous Economies," Working paper, ETH Zurich.

[37] Lettau, M. and J. Wachter (2009), "The Term Structures of Equity and Interest Rates," forthcoming, Journal of Financial Economics.

[38] Longstaff, F. A. and J. Wang (2009), "Asset Pricing and the Credit Market," Working Paper, MIT and University of California at Los Angeles.

[39] Malmendier, U. and S. Nagel (2010), "Depression Babies: Do Macroeconomic Experiences Affect Risk-Taking?," Quarterly Journal of Economics, forthcoming.

[40] Mehra, R. and E. Prescott (1985), "The Equity Premium: A Puzzle," Journal of Monetary Economics, 15, 145161.

[41] Mehra, R. and E. Prescott (2008), Handbook of the Equity Risk Premium (Elsevier B.V.).

[42] Negishi, Takashi (1960), "Welfare Economics and Existence of an Equilibrium for a Competitive Economy," Metroeconomica, 12, 92-97.

[43] Nualart, David (2006), "The Malliavin calculus and related topics. Probability and its Applications," Second edition ed., Berlin: Springer-Verlag.

[44] Paravisini, D., V. Rappoport, and E. Ravina (2010) "Risk Aversion and Wealth: Evidence from Person-to-Person Lending Portfolios, Working paper, Columbia Business School.

[45] Sandmo, A. (1970), "The Effect of Uncertainty on Saving Decisions," Review of Economic Studies, 37, 353-360.

[46] Sandroni, A. (2000), "Do markets favor agents able to make accurate predictions?," Econometrica, 68, 1303-1341.

[47] Shiller, R. J. (1981), "Do Stock Prices Move Too Much to be Justified by Subsequent Changes in Dividends?," American Economic Review, 71, 421-436.

[48] Wang, Jiang (1996), "The Term Structure of Interest Rates in a Pure Exchange Economy with Heterogeneous Investors," Journal of Financial Economics, 41 (1), 75-110. 
[49] Weil, P. (1989), "The Equity Premium Puzzle and the Risk-Free Rate Puzzle," Journal of Monetary Economics, 24, 401421.

[50] Wilson, R. B. (1968), "The Theory of Syndicates," Econometrica, 36, 119-132.

[51] Yan, Hongjun (2008), "Natural Selection in Financial Markets: Does It Work?," Management Science, 54 (11), 1935-1950.

[52] Zapatero, F. (1998), "Effect of Financial Innovations on Market Volatility when Beliefs are Heterogeneous," Journal of Economic Dynamics and Control, 22, 597-626. 